\title{
Des architectes japonais en France. Les parcours de Ban Shigeru, Kuma Kengo, Fujimoto Sou et Tane Tsuyoshi
}

\author{
海外の日本人建築家たち：坂茂、隈研吾、藤本壮介、田根剛のフラ \\ ンスの建築事務所の設立における戦略
}

Japanese Architects in France: An Analysis of the Works of Ban Shigeru, Kuma Kengo, Fujimoto Sou and Tane Tsuyoshi

Salvator-John A. Liotta et Aya Jazaierly

\section{OpenEdition} Journals

\section{Édition électronique}

URL : https://journals.openedition.org/ebisu/5275

DOI : $10.4000 /$ ebisu. 5275

ISSN : 2189-1893

\section{Éditeur}

Institut français de recherche sur le Japon à la Maison franco-japonaise (UMIFRE 19 MEAE-CNRS)

\section{Édition imprimée}

Date de publication : 15 décembre 2020

Pagination : 267-324

ISSN : 1340-3656

\section{Référence électronique}

Salvator-John A. Liotta et Aya Jazaierly, « Des architectes japonais en France. Les parcours de Ban Shigeru, Kuma Kengo, Fujimoto Sou et Tane Tsuyoshi », Ebisu [En ligne], 57 | 2020, mis en ligne le 20 décembre 2020, consulté le 21 septembre 2021. URL : http://journals.openedition.org/ebisu/5275 ; DOI : https://doi.org/10.4000/ebisu.5275 


\section{Des architectes japonais en France : les parcours de Ban Shigeru, Kuma Kengo, Fujimoto Sou et Tane Tsuyoshi Salvator-John A. Liotta \& Aya JazaierLy}

海外の日本人建築家たち：坂茂、隈研吾、藤本壮介、田根剛のフランスの建築事務所

の設立における戦略

サルヴァトール＝ジョン・リオッタ、アヤ・ジャゼルリー

Japanese Architects in France: An Analysis of the Works of Ban Shigeru, Kuma Kengo, Fujimoto Sou and Tane Tsuyoshi

Salvator-John LiotTa \& Aya JAZAierLY

\ Mots-clés : agences d'architecture, architectes japonais, architecture japonaise, Ban Shigeru, Fujimoto Sou, Kuma Kengo, profession d'architecte, Tane Tsuyoshi

Les auteurs : Salvator-John A. Liotta, après un doctorat au Japon et une expérience en tant qu'architecte et chercheur auprès de Kuma Kengo, est professeur associé à la faculté d'architecture La Cambre-Horta de l'université Libre de Bruxelles, associé au bureau LAPS Architecture et correspondant de la revue d'architecture DOMUS.

Aya Jazaierly, après un doctorat à l'université de Tokyo et une expérience professionnelle dans l'agence d'Isozaki Arata, gère depuis 2017 le cabinet Maru Architecture et enseigne aux universités américaine et arabe de Beyrouth.
Résumé : Grâce à une présentation de l'approche conceptuelle et de la pratique professionnelle de quatre architectes japonais dans le cadre de leurs agences implantées en France, nous souhaitons analyser les contextes contemporains de la profession d'architecte dans les deux pays. Quelles sont les raisons qui les incitent à travailler hors du Japon ? Pourquoi le marché français permet-il aux architectes étrangers d'accéder aux concours publics locaux ? Nous proposons un éclairage sur cet échange franco-japonais où, d'une part, ces architectes japonais s'adaptent au contexte de la construction française et, d'autre part, la création architecturale en France élargit son rayonnement international. 


$$
\text { キーワード }
$$

建築事務所、建築家、日本人建築家、日本の 建築、隈研吾、坂茂、田根剛、藤本壮介

\section{著者}

サルヴァトール＝ジョン・リオッ夕：日本で 博士号を取得。隈研吾に師事し、建築家や研 究者としての経験を積んだ後、ブリュッセ ル自由大学カンブル・オル夕建築学部の教授 となる。LAPS 建築事務所に所属し、建築誌 $\ulcorner$ DOMUS」の執筆も担当する。 アヤ・ジャゼルリー：東京大学にて博士号 を取得。磯崎新の事務所で経験を積んだ後、 2017 年よりマル建築事務所を設立し、ベイ
ルート・アメリカ大学とベイルート・アラブ 大学で教鞭をとる。

\section{要旨}

4 人の日本人建築家のフランスを拠点とする 建築事務所の概念的アプローチや専門的実践 を紹介し、両国における建築家の現代の状況 の分析を行う。彼らが日本以外で活動するの はなぜなのか? ?ランスの市場では、なぜ外 国人建築家がフランスの公募に参加できるの か?これらの日本人建築家がフランスの建築 事情に適応し、フランスの創造建築の影響が 国際的に広がる日仏の交流に焦点を当てる。
Keywords: Architectural Profession, Architecture Offices, Ban Shigeru, Fujimoto Sou, Kuma Kengo, Japanese Architects, Japanese Architecture, Tane Tsuyoshi

The Authors: Salvator-John A. Liotta is an associate professor at Université Libre de Bruxelles, Faculty of Architecture La Cambre-Horta, a partner of LAPS-Architecture office, and a long-time correspondent of Domus magazine.

Aya Jazaierly, after a PhD from the University of Tokyo, has been managing her practice Maru Architecture since 2017, and is currently an adjunct professor at the American University of Beirut and at the Beirut Arab University (RIBA accredited).

\begin{abstract}
By introducing the philosophy and practice of four Japanese architects in their establishment in France, we aim to analyse the contemporary contexts of the architectural profession in both countries. What are the reasons behind the Japanese architects' eagerness to work outside Japan? Why does the openness of the French market allow international architects to access French public competitions? We would like to emphasise the result of this exchange, where, on one hand, Japanese architects are adapting to the French construction context, and, on the other, the French architectural milieu is expanding its international exposure.
\end{abstract}




\title{
Des architectes japonais en France. Les parcours de Ban Shigeru, Kuma Kengo, Fujimoto Sou et Tane Tsuyoshi
}

\author{
Salvator-John A. LIOTTA** \& Aya JAZAIERLY***
}

\section{Introduction}

Au cours de l'ère Heisei 平成 (1989-2019), si plusieurs architectes japonais réputés conçoivent des projets à l'étranger - principalement en Asie et en Europe, et dans une moindre mesure aux États-Unis -, rares sont ceux qui y établissent un bureau car sa soutenabilité financière demeure le privilège des grandes firmes du domaine. Quelques exceptions sont pourtant notables en Europe ${ }^{1}$ tandis que la dynamique du marché asiatique va de

1. À Barcelone par exemple, avant la crise de 2008, l'ouverture culturelle puis le boom immobilier ont vu l'implantation d'annexes (minuscules) d'au moins deux agences d'architecture japonaise très médiatisées : celle d'Isozaki Arata* 磯崎新 (né en 1931) en 1985, à l'origine pour la réalisation du stade olympique Palau Sant Jordi des Jeux de 1992, puis pour d'autres projets dans différentes villes du pays (Arata Isozaki Studio BCN, dirigée par l'architecte Tange Toshiaki 丹下敏明 [né en 1948] qui précise que la municipalité l'impose dès lors qu'il y a construction du projet. Voir http://www.

* Les mots suivis d'un astérisque renvoient au lexique situé à la fin du dossier.

** Professeur associé à la faculté d'architecture La Cambre Horta, université libre de Bruxelles.

*** Docteure en architecture, architecte, enseignante à l'université américaine de Beyrouth. 
plus en plus attirer à partir des années 2000 les plus grandes signatures des «starchitectes » mondiaux, y compris japonaises. Une poignée d'architectes représentatifs de l'ère Heisei, vont tenter d'ouvrir des agences dans les métropoles asiatiques voisines alors en pleine effervescence économique ${ }^{2}$.

En dehors des effets conjoncturels, cette tendance résulte de facteurs relatifs à la globalisation, tels que l'ouverture internationale des marchés et l'internationalisation des opérateurs des secteurs de l'immobilier, de l'ingénierie et de la construction (Biau 2020 : 15), favorisées par l'introduction de nouvelles technologies de communication et la facilité croissante des déplacements. Dans le cas du Japon, d'autres facteurs sont spécifiques à la situation professionnelle propre au pays avec notamment les difficultés rencontrées par les architectes indépendants pour accéder à leur marché public local car celui-ci est dominé par de puissantes entreprises de construction ou de grands bureaux d'étude. De plus, selon Itō Toyō, lauréat du prix Pritzker* en 2013, il existe aussi un problème de statut et de reconnaissance de l'architecte dans la société japonaise :

Le statut de l'architecte dans la société japonaise diffère de celui de l'architecte aux États-Unis ou en Europe. Dans le cas des États-Unis, où l'économie de marché est encore plus mondialisée qu'au Japon, si l'architecte n'est pas dans le système, il lui est impossible de travailler. L'architecture est évaluée de la même façon que les œuvres d'art. On ne juge pas de sa valeur sociale, mais de l'originalité de l'architecte, que l'on achète contre de l'argent. De ce fait, des architectes comme Frank O. Gehry, capables de concevoir des bâtiments extrêmement originaux, semblables à des œuvres d'art, sont très appréciés. On fait venir des architectes célèbres du monde entier pour leur commander des bâtiments. On donne ainsi de la valeur aux projets, on acquiert un certain statut, voilà comment les choses fonctionnent. En revanche, dans la société européenne, les commandes publiques sont au cœur du travail des architectes. [...] Au Japon, de l'après-guerre jusqu'aux années 1960 environ, comme en Europe, l'architecte considérait que son travail était de bâtir des édifices publics

spaceunder.com/editorial/2013/jun/6/arata-isozaki-visit-bcn-studio/ et un entretien de 2012 : https://www.youtube.com/watch?v=UtWgDs1nzsl), et celle d'Itō Toyō* 伊東豊雄 (né en 1941) plus tard en 2006 (Toyo Ito Arquitectos Asociados en Espana) en lien avec plusieurs programmes prestigieux réalisés dans la capitale catalane.

2. Par exemple : Takamatsu Shin* 高松伸 (né en 1948) (Taipei, Hanoi), Yamamoto Riken* 山本理顕 (né en 1945) (Pékin), Kuma Kengo* 隈研吾 (né en 1954) (Shanghai, Pékin). 
pour la population. À l'époque, les architectes et la société entretenaient une relation saine. (Itō 2014 : 143-144)

Aujourd'hui, cette relation a évolué et les jeunes architectes japonais sont réputés pour concevoir des maisons individuelles de manière créative, ce qui est loué par la presse spécialisée nationale et internationale. Mais cette créativité est considérée par Itō comme un signe de frustration qui reflète l'impossibilité des architectes japonais indépendants à accéder à leur marché national et à réaliser des bâtiments publics. Elle a paradoxalement abouti à l'émergence d'une "nouvelle " architecture japonaise, fortement reconnue en dehors du pays. Ce qu'Itō confirme : "L'architecte [japonais] indépendant, puisqu'il n’est pas sollicité pour les projets publics, est évidemment frustré. Il évacue donc cette frustration en investissant toute son énergie dans des maisons individuelles et propose une architecture novatrice où la recherche esthétique a remplacé la critique. Et cette originalité fait alors grimper sa cote à l'étranger. " (Itō 2014 : 145). À titre d'illustration, ArchDaily - l'un des sites Internet les plus consultés au monde par les architectes - a collecté près de 1600 projets japonais depuis 2008 : 60\% d'entre eux concernent des maisons individuelles, dont les pages sont les plus visitées et les préférées des internautes (Kofler 2017 : 15).

Dans ce contexte, la France apparaît comme l'une des destinations privilégiées par les architectes japonais du fait d'un environnement législatif particulier lié à la loi sur l'architecture instaurée en $1977^{3}$, ainsi qu'au système d'appel d'offres public établi par la loi relative à la maitrise d'ouvrage publique (dite loi MOP) en 1985. Ce cadre fait de la France un marché attractif pour les bureaux d'architecture indépendants ${ }^{4}$.

3. Dans son article "Pourquoi une loi sur l'architecture?"(2017), Julien Giusti, chargé de mission à la direction de l'Architecture (ministère de la Culture) de 1976 à 1980, explique les étapes qui ont mené vers une politique de la qualité architecturale et urbaine qui aboutira à cette loi sur l'architecture.

4. Les nombreux concours internationaux organisés en France durant les années 1980 ont attiré plusieurs architectes japonais. On relève 21 participants japonais pour le concours des Halles (1975), puis 58 pour celui du Parc de La Villette (1982), 12 pour la Tête-Défense (1982), 27 pour l'Opéra Bastille (1983), et 217 pour la Maison de la culture du Japon à Paris (1989), tous situés dans la capitale. Les années 1990 ont vu quant à elles la construction à Paris de projets conçus par des architectes japonais célèbres : immeuble Grand Écran sur la place d'Italie (1992), Tange Kenzō* 丹 下健三 (1913-2005); tour Pacifique (1992) à la Défense, Kurokawa Kishō* 黒川紀章 
Ce constat soulève plusieurs questions : Pourquoi des architectes japonais décident-ils de travailler à l'étranger? Pourquoi certains d'entre eux ont-ils implanté un bureau en France? Comment le contexte français influence-t-il leurs processus de création et de conception? En quoi ces projets se distinguent-ils de ce qu'ils construisent au Japon ou ailleurs?

Afin de répondre à ces questions, cet article se concentre sur les expériences de quatre architectes japonais en France: Ban Shigeru, Kuma Kengo, Fujimoto Sōsuke (Sou)* 藤本壮介 (né en 1971) et Tane Tsuyoshi* 田根剛 (né en 1979). Il explore les différentes raisons qui les ont conduits à s'implanter dans ce pays, leurs stratégies, leurs méthodes, leurs styles et leurs réalisations, alors que d'autres architectes japonais ont travaillé en France sans y établir d'agence, comme par exemple Andō Tadao, SANAA, Itō Toyō ou Atelier Bow-Wow. Les quatre architectes choisis présentent des parcours très différents, ce qui permet de montrer une certaine pluralité de situations. Ban Shigeru a démarré son activité professionnelle en France grâce à un concours sur invitation tandis que Kuma a gagné deux concours ouverts dans le cadre du système français. Fujimoto est entré sur le marché français en remportant un concours public pour un ensemble résidentiel qui exigeait l'association d'un jeune cabinet français avec un architecte de renommée internationale. Tane, quant à lui, était déjà en France lorsqu'il a décidé d'y établir son propre bureau.

Cette étude se base sur les statistiques établies par le Conseil national de l'Ordre des architectes (CNOA) en France. Elle s'inscrit dans le cadre d'une recherche plus vaste qui examine les données relatives à la profession d'architecte et au marché français de la construction. En plus de données

(1934-2007); Espace de méditation de l’Unesco (1995), Andō Tadaō* 安藤忠雄 (né en 1941). Dans la première décennie du $\mathrm{XxI}^{\mathrm{e}}$ siècle, des architectes comme Itō Toyō, Ban Shigeru* 坂茂 (né en 1957), Atelier Bow-Wow* [Atorie Wan アトリエワン], Kuma Kengo et l'agence SANAA* sont également intervenus à Paris et ailleurs en France. Itō Toyō a conçu le comptoir de la Japan Airlines (1991) et le nouvel hôpital Cognacq-Jay à Paris (2006); Ban Shigeru le Centre Pompidou de Metz (2010) et la Seine Musicale à Boulogne-Billancourt (2017); Atelier Bow-Wow, les logements Autrement rue Pierre Rebière à Paris (2012); Kuma Kengo la boutique de thé Jugetsudo à Paris (2008), la restructuration du siège social de Sysla à Paris (2010), la Cité des Arts de Besançon (2013), le Fonds régional d'art contemporain (FRAC) de Marseille (2013), le Pôle multi-équipements Macdonald à Paris (2014); SANAA le musée Louvre-Lens (2012) et la restructuration du magasin La Samaritaine à Paris (2020). 
statistiques, nous nous sommes également appuyés sur des entretiens avec les quatre architectes choisis, entre mai 2017 et décembre 2018, qui nous ont permis d'établir différentes comparaisons.

\section{Le milieu architectural professionnel au Japon et en France}

\section{1. La profession d'architecte au Japon et} le rôle des entreprises générales de construction

La profession d'architecte a été introduite au Japon pendant l'ère Meiji 明治 (1868-1912), remettant en question les pratiques traditionnelles et anciennes de la construction en bois ainsi que les divers métiers de la construction qui l'entourent (Jacquet, Matsuzaki \& Tardits 2019). Les maîtres charpentiers ont dû relever le défi de s'adapter aux exigences et au rythme d'une nouvelle société en plein bouleversement et développement, en élargissant leur savoir-faire à l'utilisation de l'acier et de la brique (Reynolds 2004; Frampton \& Kudo 1997). Ce sont ces corporations de charpentiers qui ont fondé des entreprises générales de construction, dénommées par la suite zenekon* ゼネコン; les plus importantes sont actuellement connues sous le nom de Big Five : Kajima kensetsu 鹿島建設 (1840), Shimizu kensetsu 清水建設 (1804), Taisei kensetsu 大成建設 (1873), Ōbayashi gumi 大林組 (1892) et Takenaka kōmuten* 竹中工務店 $(1899)^{5}$. Celles-ci contrôlent un tiers du marché de la construction au Japon (Steele 2017 : 46; Bognar et al. 2000). Ces cinq entreprises s'inscrivent toutes dans la continuité d'une longue tradition de charpenterie qui a soutenu les transformations des pratiques de construction en intégrant de nouvelles méthodes d'ingénierie et l'emploi de matériaux et techniques modernes. Leurs possibilités et capacités vont au-delà de celles habituellement dévolues à l'architecte-concepteur et englobent celles du constructeur.

Parallèlement, et aussi pendant l'ère Meiji, d'importants bureaux d'étude ont développé de larges compétences en architecture, urbanisme et ingénierie mises au service de la modernisation de la société japonaise. Sur cette base, et dans la continuité de la tradition constructive issue de la charpenterie, deux catégories principales opèrent actuellement dans le secteur du

5. Voir la notice «Zenekon» dans Bonnin, Nishida \& Inaga (2014 : 543). 
bâtiment au Japon : d'une part, les entreprises générales de construction incluant les "fabricants de maisons ${ }^{6}$ " et, d'autre part, les bureaux d'étude en construction et en architecture en tant que prestataires de services. Cette dernière catégorie comprend de grandes entreprises employant des centaines d'architectes et d'ingénieurs telles que Nikken sekkei* 日建設計 qui recourt à plus de 1300 architectes (Nikken sekkei 2019), ou Nihon sekkei 日本設計, à côté de petites agences - dénommées atorie* アトリエ employant moins de trente architectes, telles que les agences d'Isozaki Arata, SANAA, Yamamoto Riken par exemple (Centre de coopération industrielle UE-Japon 2015).

Les Big Five emploient plus de dix mille architectes; Takenaka kōmuten emploie à elle seule plus de deux mille architectes certifiés. Plaçant la collaboration et la recherche au centre de leurs valeurs et de leurs pratiques, les bureaux d'étude de ces zenekon sont reconnus capables d'un design de haute qualité et d'une excellente maîtrise des coûts et du calendrier de livraison des chantiers (Frampton \& Kudo 1997). Dans le même registre, les entreprises de construction de maisons - pour la plupart fondées au cours de la seconde moitié du $\mathrm{Xx}^{\mathrm{e}}$ siècle - se sont spécialisées dans la conception et la construction de logements individuels au Japon. Daiwa House [Hausu] 大和ハウス et Sekisui House [Hausu] 積水ハウス, les deux plus grandes, emploient plus de 15000 personnes. Ces entreprises conçoivent, construisent et vendent des maisons prêtes à habiter en fournissant des certificats de performance, des tests sismiques et des contrats d'entretien inclus dans les prestations (Barr 2017; The Japan Institute of Architects 2014-2015).

Dans ce panorama, il existe aussi près de 3000 bureaux d'étude (architecture et ingénierie) indépendants qui obtiennent 10 à $15 \%$ du total des contrats publics et privés au Japon. $80 \%$ de cette part sont captés par de grands bureaux d'étude - une centaine de zenekon et de grandes entreprises - tandis que les $20 \%$ restants sont partagés entre les 2900 autres bureaux, dont les agences d'architecture (Centre de coopération industrielle UE-Japon 2015).

Dans ce contexte, les architectes nouvellement établis ont du mal à accéder au marché local de la construction. En ce qui concerne les maisons

6. Appelé en japonais hausu mēkā ハウスメーカー (de l'anglais house maker, parfois une filiale de zenekon). 
individuelles, seulement $2 \%$ sont conçues par un atelier d'architecture, car le propriétaire d'un terrain préfère avoir recours à un constructeur spécialisé qui fournit un projet et un bâtiment clés en main avec des garanties de sécurité (Nuijsink 2012).

Chaque année, plus de 12000 étudiants sortent diplômés de l'une des 130 facultés d'architecture du pays. La plupart de ces diplômés sont embauchés par des entreprises de construction ou par les administrations. Pour obtenir la certification d'architecte de première ou de deuxième classe (soit la licence d'exercice, et acquérir ainsi le titre de kenchikushi 建築士), un architecte doit, en plus de son diplôme en architecture, passer par un processus d'examens et d'évaluations. Le nombre total de kenchikushi dépasse actuellement les 500000 (Architectural Institute of Japan 2019).

\section{2. La profession d'architecte en France et la prédominance des petites agences d'architecture}

En France, depuis 1977, le marché de la construction est réglementé par la loi $\mathrm{n}^{\circ} 77-2$ qui vise à impliquer les architectes et promeut l'architecture par le biais de concours pour la construction de nouveaux bâtiments publics. La loi affirme que « l'architecture est une expression de la culture et qu'en conséquence, la création architecturale, la qualité des constructions, leur insertion dans le milieu environnant, le respect du paysage naturel et urbain ainsi que du patrimoine sont d'intérêt public». Cette loi a été plusieurs fois amendée depuis 1977 et impose l'organisation d'un concours d'architecture si le montant des travaux dépasse 90000 euros ou si la surface à bâtir est supérieure à 2500 mètres carrés ${ }^{7}$. Une annonce doit être publiée sur diverses plateformes publiques afin d'assurer une concurrence transparente et impartiale. Il est important de noter que les participants retenus reçoivent un forfait censé couvrir les coûts de base liés au travail requis pour le concours (JORF 2018). Cela permet à de petites agences d'architecture d'orienter leur énergie et leurs ressources vers la dimension créative de la conception. Dans les concours internationaux - à l'exception des concours prestigieux restreints -, aucune rémunération n'est versée aux architectes

7. Voir le Journal officiel de la République française, nº 50, 28 février 2017. 
participants. La loi sur l'architecture donne la priorité à la qualité des constructions sur la production de masse (surtout des logements).

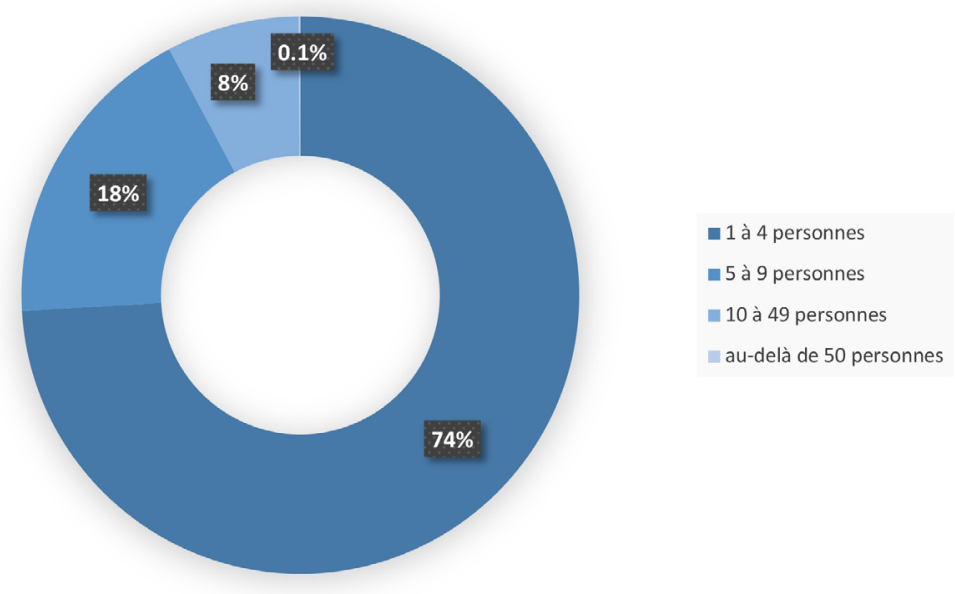

Fig. 01

Les agences d'architecture en France : répartition par taille.

Réalisation : les auteurs. Source : Mirza \& Nacey Research (2018).

Comme le montre la figure 01 ci-dessus, le paysage français des agences d'architecture est dominé par de très petites structures $(74,1 \%)$ qui emploient au plus quatre personnes ${ }^{8}$. Parallèlement, seul $0,1 \%$ des bureaux français emploie plus de 50 personnes, comme par exemple les Ateliers Jean Nouvel (AJN) avec plus de 120 personnes (AJN 2019). L'exercice individuel, à titre libéral, constitue le gros de la profession en France ${ }^{9}$. En 2018, 80 \% des entreprises du secteur ont un effectif inférieur

8. En 2018, environ 30000 architectes professionnels travaillent en France dans 8033 agences enregistrées (Mirza \& Nacey Research 2018 : 79).

9. Le rapport Archigraphie de 2020 constate qu'en France, les 29034 architectes inscrits à l'Ordre se répartissent ainsi : "En 2019, on compte 12399 architectes libéraux, 13773 architectes associés, 855 salariés, 912 fonctionnaires, 699 architectes n'exerçant pas la maîtrise d'œuvre et 396 architectes exerçant exclusivement à l'étranger » (Ordre des architectes, France, $2020: 38$ ). 
à cinq ETP (équivalent temps plein). Compte tenu de cette situation, la majorité des agences d'architecture françaises ont une taille qui ne leur permet pas d'être compétitives à l'étranger face à de grandes structures telles que Foster \& Partners ou Zaha Hadid* Architects au Royaume-Uni - la première employant 1183 personnes et la seconde 312 en $2012^{10}$-, ou bien Skidmore Owings and Merrill (SOM) et Kohn Pedersen Fox (KPF) aux États-Unis, cette dernière employant plus de 570 personnes en 2017 (Shikata et al. 2017). Ainsi, à peine $5 \%$ du chiffre d'affaires de l'architecture française est réalisé à l'étranger (Ordre des architectes, France, 2018).

Une particularité de la loi française est d'avoir ouvert la porte aux agences non seulement françaises mais aussi étrangères, leur permettant de postuler et participer à des concours pour des programmes en France. Face à l'absence d'agences d'architecture de moyenne ou grande taille, des architectes étrangers proposent leurs services sur le marché français (Ordre des architectes, France, 2018). De 1983 à 2009, on passe de 617 à 1922 architectes étrangers qui ont laissé une trace de leur passage en France, comptant pour, respectivement, de $3,7 \%$ à $6,8 \%$ du nombre total des architectes inscrits. En 2019, le nombre d'architectes étrangers inscrits à l'Ordre national des architectes s'élève à 1870 sur un total de 29900 membres, soit un pourcentage de $6,2 \%{ }^{11}$. Les deux tiers sont des ressortissants de l'Union européenne et un tiers vient de pays comme l'Algérie, le Cameroun, la Chine, la Corée du Sud, l'Iran et le Maroc (Nogue 2010). Certains observateurs y voient " le mécanisme de la libre concurrence européenne qui est à l'œuvre " mais aussi le résultat d'une "stratégie offensive de marketing et de lobbying pour pénétrer le marché français » (Lemoine 2019 :101).

Ces différents facteurs - la loi sur l'architecture, la libre concurrence des marchés publics, le manque de commandes publiques et privées pour les jeunes architectes au Japon et l'émergence d'un engouement pour " l'architecture japonaise "- ont poussé des architectes japonais à rechercher des opportunités en France, jusqu'à s'y implanter. Les quatre études de cas suivantes clarifient les raisons et modalités de la présence professionnelle de ces architectes japonais qui ont ouvert un bureau à Paris.

10. D’après Lago-Novás Domingo J. (2014).

11. Basé sur les données fournies par l'Ordre national des Architectes en France, en date du 21 août 2019. 


\section{Ban Shigeru : « ne pas être japonais ", une stratégie pour travailler à l'étranger}

Ban Shigeru est parti faire ses études à l'Institut d'architecture de Californie du Sud (SCI-ARC) puis est entré en 1980 à l'école d'art et d'architecture Cooper Union à New York, où il a étudié sous la direction de John Hejduk $^{12}$ (1929-2000) avant d'obtenir son diplôme en 1982 (Shigeru Ban Architects 2020). Il s'est progressivement engagé dans un processus expérimental d'étude des matériaux ainsi que de leur expression structurelle et architecturale. Il s'est concentré sur ce qu'il appelle la structure invisible, qui évite les éléments structurels excessifs et vise à les fondre dans la forme tridimensionnelle globale du bâtiment (Jodidio 2016).

Ban est connu pour son travail novateur employant du papier et des tubes en carton recyclés, ainsi que pour son engagement dans des activités humanitaires sur des sites touchés par des catastrophes, pour lesquels il a reçu en 2014 le $37^{\mathrm{e}}$ prix Pritzker. Aujourd'hui, il dirige trois bureaux : à Tokyo dès 1987, à Paris depuis 2004 et à New York depuis 2005.

Parmi ses œuvres les plus remarquables, on peut citer le Pavillon japonais (2000) à l'Exposition universelle de Hanovre, le Centre Pompidou-Metz (2010), le musée d'Art d'Aspen (2014) aux États-Unis (fig. 02) et La Seine Musicale (2017), un complexe culturel avec des salles de concert sur l'Île Seguin à Boulogne-Billancourt.

12. L'architecte américain John Hejduk y instaure à partir des années 1960 un enseignement centré sur l'architecture comme discipline autonome et soulignant l'importance du processus de conception en tant qu'expérience intellectuelle. 


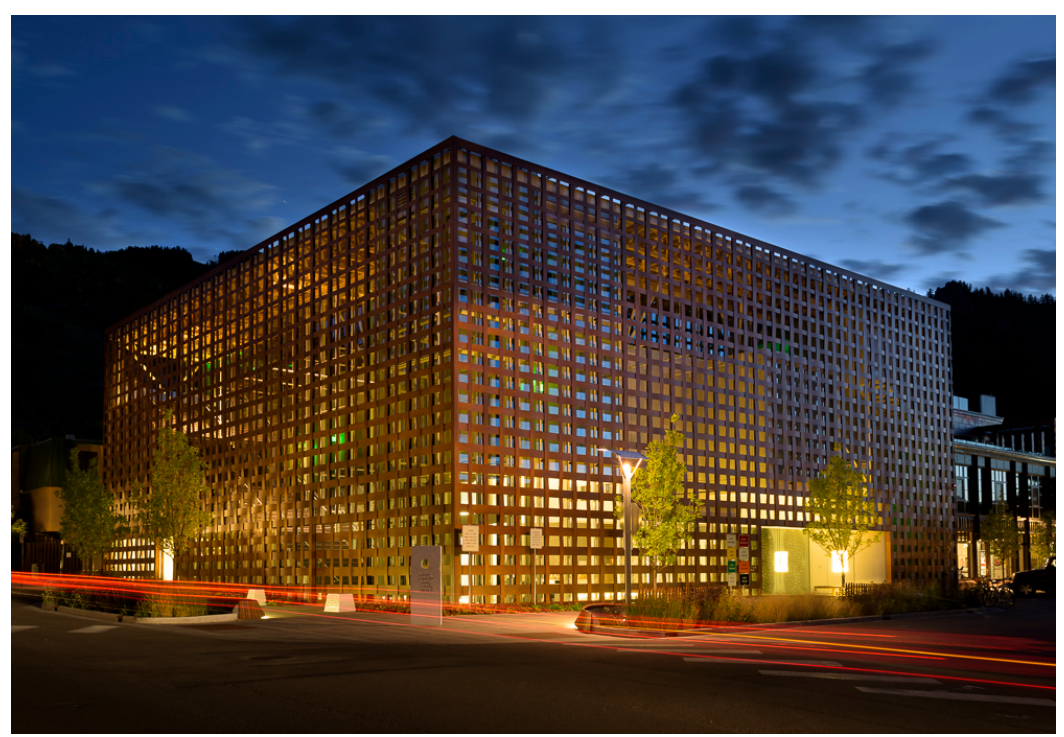

Fig. 02

Musée d'Art d'Aspen, États-Unis, 2014, par Ban Shigeru.

(c) Bkthomson 16 .

\section{1. Ouverture sur la scène internationale}

À l'inverse d'autres architectes japonais, Ban ne met pas en avant ses origines comme instrument principal pour obtenir de nouvelles commandes.

Il se peut que je sois inconsciemment influencé par la culture japonaise, mais en général je n'aime pas que les critiques relient mon architecture à la culture japonaise. Il y a plusieurs architectes japonais qui utilisent leur japonité comme stratégie pour gagner des concours et faire de l'architecture. Ce n'est pas mon cas, par exemple pour la Maison Curtain Walls (Tokyo, 1995), une de mes premières œuvres, j’ai été fortement influencé par des maisons en Californie que j'ai visitées quand j'ai commencé à étudier l'architecture aux États-Unis. Je me souviens qu'après être allé à Beyrouth, j'ai été surpris de voir combien de balcons ont de grands rideaux ombrageant, dans un sens c'est très similaire à l'un de mes concepts ${ }^{13}$.

13. Toutes les citations de Ban Shigeru sont des transcriptions d'un entretien enregistré et traduit de l'anglais au français par les auteurs en mai 2017. 
Ban a décidé d'ouvrir un bureau à Paris en 2004 après avoir remporté le concours pour le Centre Pompidou à Metz (fig. 03). En général, lorsque les architectes gagnent un concours à l'étranger, ils engagent des architectes locaux pour prendre en charge sur place les phases de conception, de développement et d'exécution. Cependant, Ban affirme qu'il a fait tout son possible pour éviter ce scénario car la relation entre les architectes étrangers et locaux peut devenir problématique. Il estime que pour contrôler et garantir la conception originale jusqu'à l'achèvement, il est important de maintenir une communication directe entre le maitre d'œuvre et le commanditaire. Pour Ban, il est risqué de laisser la responsabilité du projet à l'architecte local car ce dernier serait enclin à satisfaire les besoins du maître d'ouvrage et par conséquent pourrait compromettre la qualité de la conception :

S'il [l'architecte local] suit les idées de l'architecte mandataire, le budget et la durée d'exécution augmentent en général. Et comme l'architecte local doit côtoyer le client local pendant les cinq à dix prochaines années, il lui est très important de satisfaire le client au lieu d'aider à réaliser les demandes de l'architecte mandataire. C'est pourquoi, souvent, l'architecte local - qui est censé être un partenaire - peut devenir un ennemi potentiel.

Ban a considéré le projet de Metz comme extrêmement important pour sa carrière d'architecte, raison pour laquelle il a tenu à en perfectionner la conception et à éviter toute erreur : " Je voulais contrôler tout le processus moi-même, c'est pourquoi j'ai ouvert un bureau à Paris » qui, au début et pendant la période nécessaire à la livraison du projet, était hébergé dans une structure temporaire construite sur les terrasses du Centre Pompidou à Paris.

Selon lui, exercer en France est à la fois passionnant et stimulant car cela invite l'architecte à travailler sur des projets de grande envergure et complexes, parfois sans expérience préalable :

La France est le meilleur pays au monde pour l'architecture. Même au Japon, ou aux États-Unis où j'ai étudié, si vous n'avez pas d'expérience dans la conception de musées, vous ne serez jamais invité à participer à un concours de musée, mais en France, cela n'a pas d'importance. Quand j'ai gagné le concours pour le Centre Pompidou, je n'avais jamais conçu de musée auparavant, quand j’ai gagné le concours pour La Seine Musicale, je n’avais jamais conçu de théâtre auparavant, mais pour les clients en France, cela n'a pas d'importance. Cela n'arriverait jamais au Japon ou aux États-Unis. 
Pour ce troisième lauréat japonais du prix Pritzker, la France offre des opportunités inédites car les maitres d'ouvrage sont en attente d'idées et de créations intéressantes. En revanche, Ban considère que la phase de construction en France est la plus difficile au monde, à cause des antagonismes entre entreprises et architectes. À cet égard, le Japon est un pays beaucoup plus favorable car les entreprises de construction soutiennent les architectes tout au long du processus, leur permettant de concevoir et d'exécuter des projets de la plus haute qualité, et leur niveau de technicité est très élevé. En France, au contraire, les entrepreneurs sont en désaccord constant avec les architectes, une situation qui engendre une bataille continue entre les deux parties jusqu’à la livraison du bâtiment. Dans ce contexte, Ban insiste sur l'importance d'être présent pour « se battre » afin de maintenir la qualité de conception du projet :

Si vous engagez un architecte local, il ne sera pas assez résistant pour se battre avec les entreprises de construction que l'on trouve en France. C'est pourquoi je dois être présent tout le temps. Je fais la navette entre Tokyo et Paris chaque semaine pour cette raison. [...] Au final, je veux contrôler la conception par moi-même; c'est pourquoi je ne veux pas que mon bureau soit trop grand. 

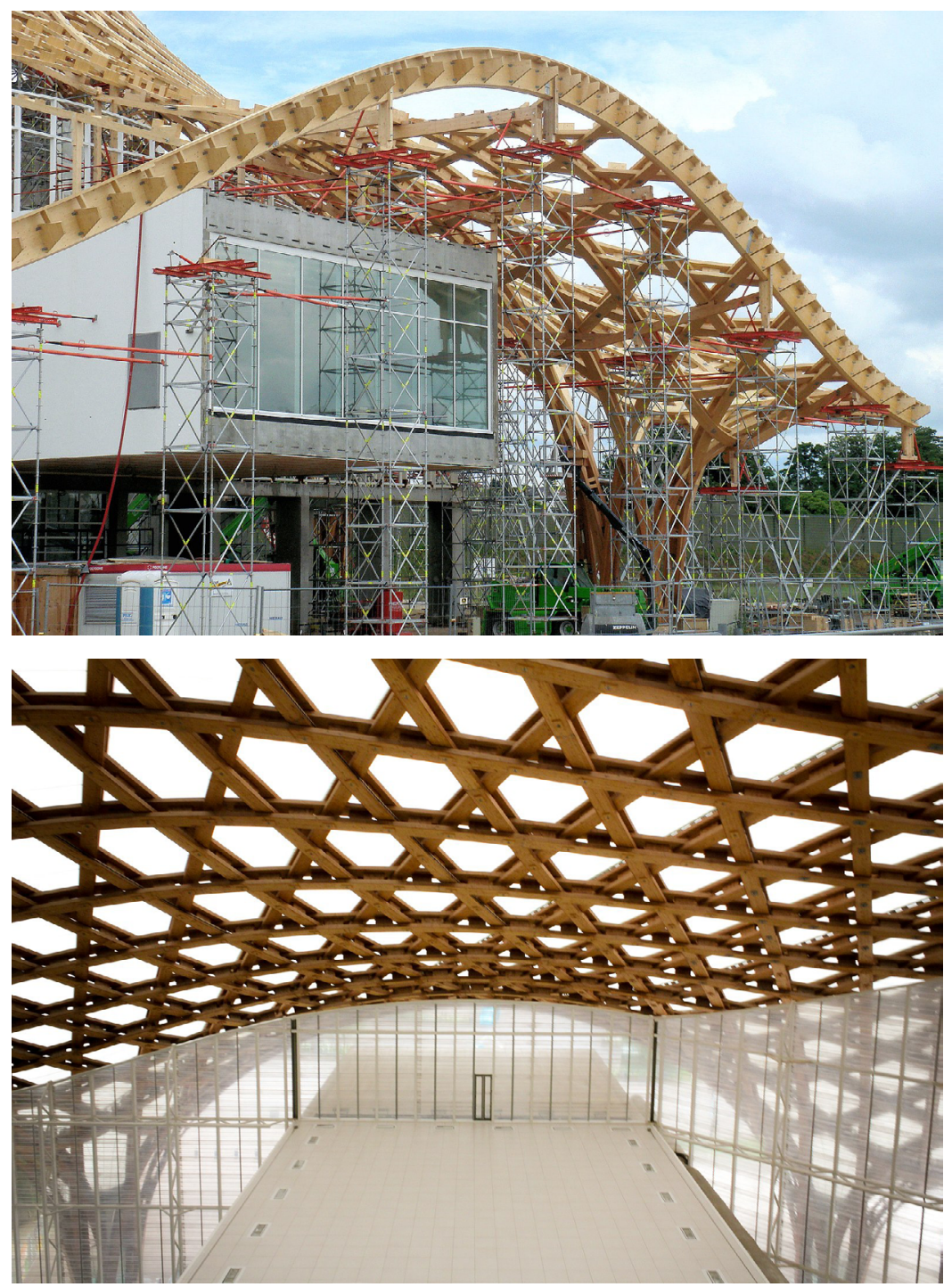

Fig. 03

Centre Pompidou-Metz.

Charpente en bois autour de la galerie 1 et pied-tulipe de la charpente, juin 2009, par Ban Shigeru.

En haut : @ MOSSOT; en bas : @ Alvarodiazsantos. 


\section{2. Différences entre le Japon, I'Europe et les États-Unis}

Travailler au Japon, aux États-Unis et en Europe offre ainsi à Ban l'opportunité d'explorer différents marchés et donc des conditions distinctes pour chaque projet. Au Japon, même si la qualité du travail des entreprises de construction est élevée, du fait des ressources limitées et de la situation géographique du pays, le nombre de spécialistes et de fabricants innovants est moindre par rapport à l'Europe. Pour lui, c'est aux États-Unis que l'architecture est la plus difficile à réaliser car la production locale limitée impose l'importation de matériaux de mauvaise qualité en provenance de la Chine ${ }^{14}$. De plus, le processus d'exécution des projets est souvent perturbé par un cercle vicieux de complications juridiques mettant en péril la réputation de l'architecte et l'achèvement des projets. Ban considère que l'Europe est l'environnement culturel, technique et juridique le plus favorable aux architectes, avec des clients soucieux de l'architecture, un nombre important d'ingénieurs et des matériaux de haute qualité fabriqués en Italie ou en Finlande. Il ajoute :

C'est pourquoi je pense que l'Europe est la région la plus propice pour faire une architecture intéressante. Lorsque j’ai ouvert mon bureau à Paris, j’ai fait venir du personnel de Tokyo, après un certain temps j'ai embauché localement. J'ai aussi un très bon partenaire français : Jean de Gastines [né en 1957]. Je me sens très chanceux.

Aujourd'hui, Ban emploie moins de vingt architectes dans son bureau français et le même nombre à Tokyo et à New York. En s'associant avec un partenaire en France et aux États-Unis, il a pu limiter le nombre de salariés en travaillant avec le même architecte local ${ }^{15}$.

\section{Kuma Kengo : adapter les stratégies à un nouveau contexte}

Kuma Kengo est professeur dans le département d'architecture de la faculté d'ingénierie de l'université de Tokyo où il dirige divers projets de recherche concernant l'architecture et le design urbain. Il est non seulement un architecte productif mais aussi un auteur prolifique, qui a développé son

14. Tel qu'exprimé par Ban lors de l'entretien avec les auteurs.

15. Depuis 1999, Jean de Gastines est son partenaire en France et Dean Maltz son partenaire aux États-Unis (www.shigerubanarchitects.com 2020). 
architecture à partir de recherches menées avec les étudiants de son laboratoire, d'essais et d'ouvrages. En 1987, il fonde à Tokyo un premier atelier dénommé Spatial Design Studio, et en 1990, il crée sa propre agence Kengo Kuma \& Associates (KKAA). Il a enseigné à l'université Columbia à New York et à l'université Keiō à Tokyo, où il a obtenu en 2008 un doctorat en architecture. Son agence emploie plus de 180 architectes à Tokyo, 30 à Paris et 20 à Pékin ${ }^{16}$. KKAA a mené des projets de musées, de salles de concert, d'hôtels, de maisons individuelles, de restaurants, de boutiques et d'équipements publics. Parmi les projets les plus reconnus, citons Aore, l'hôtel de ville de Nagaoka (2012) dans le département de Niigata, l'office du tourisme d'Asakusa à Tokyo (2012), en France la Cité des arts et de la culture à Besançon (2013, fig. 04a et 04b), en Écosse le Victoria \& Albert Museum (2018). Fin 2015, KKAA s'est vu attribuer le projet du nouveau stade olympique de Tokyo, achevé en 2020.

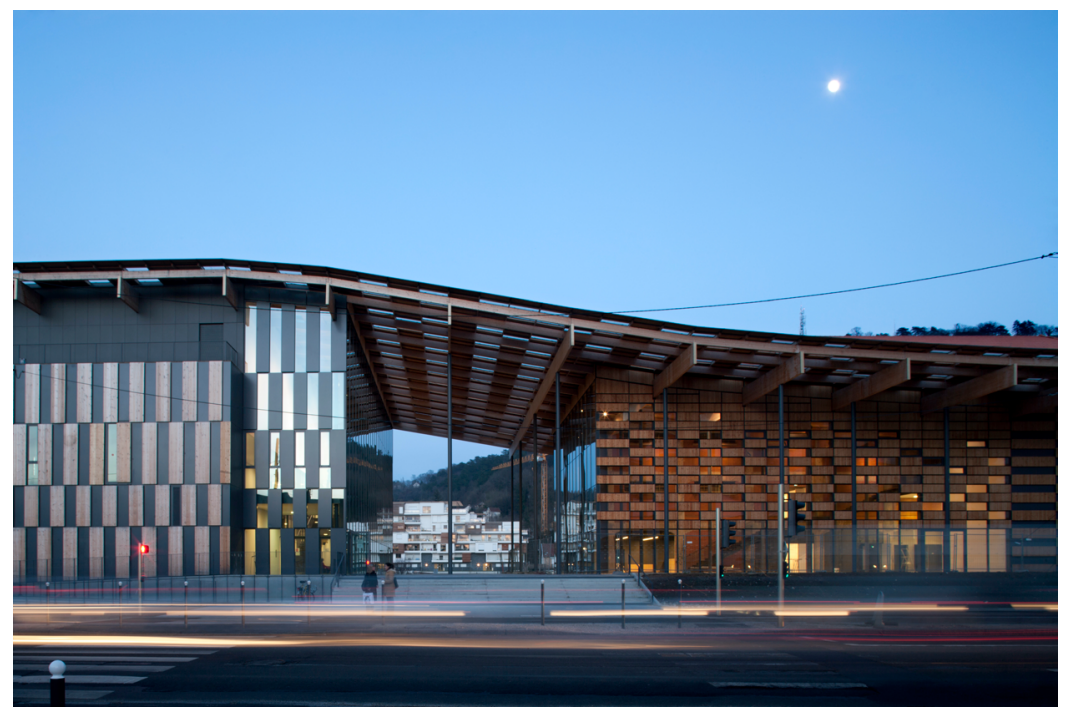

Fig. 04a

Cité des arts et de la culture à Besançon, France, 2013, par Kuma Kengo.

16. Cela correspond au nombre d'architectes professionnels, indépendamment des autres membres du personnel administratif et des stagiaires. 


\section{Fig. 04b}

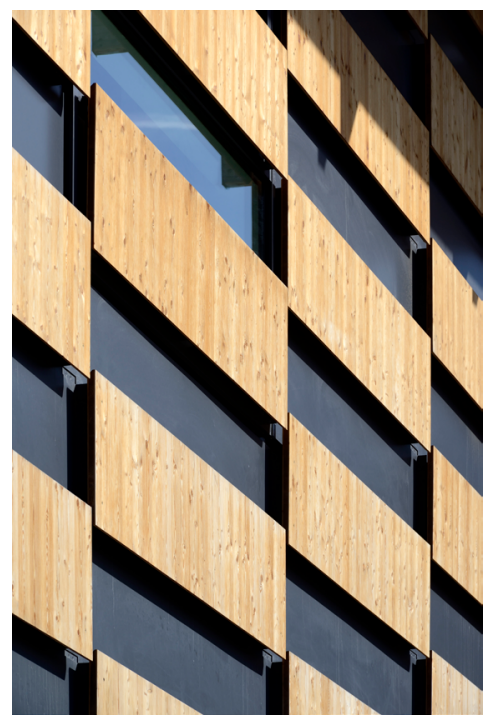

Cité des arts et de la culture à Besançon, France, 2013, par Kuma Kengo.

Photos publiées avec la permission de Kengo Kuma \& Associates Paris. Voir le numéro d'octobre 2017 de Shinkenchiku 新建築 (Nouvelle architecture).

(c) Nicolas Waltefaugle.

\section{1. Relier la nature et les gens}

L'essence de la pensée de Kuma réside dans l'importance accordée à une architecture qui relie les êtres humains à leur environnement et à la nature (Kuma 2012; Bognar 2009), et qui expérimente les matériaux plutôt que les formes. Kuma établit un langage contemporain combinant l'architecture vernaculaire et l'artisanat de la construction traditionnelle avec des technologies avancées. Son travail, immédiatement reconnaissable mais toujours renouvelé, révèle un véritable intérêt pour les techniques de construction locales mais aussi un attrait pour une architecture internationale qui cultive toutefois une attention au contexte local, à ses ressources et à son esthétique vernaculaire.

L'architecture de Kuma a connu plusieurs phases : la période dite postmoderne pendant la bulle économique japonaise des années 1980; après son éclatement, celle qualifiée d'" anti-objet" (durant les années 1990 
et le début des années 2000); et plus récemment, la période dite " organique $^{17} »$. Son travail issu de la première période présente un caractère iconique marqué lorsqu’il expérimentait les idées formelles du postmodernisme en architecture. Durant la phase " anti-objet ", Kuma s'est concentré sur « l'effacement de l'architecture », un concept concrétisé par la " fusion » du projet dans le site ou par l'intégration de matériaux naturels. La période actuelle, dite "organique", présente une architecture qui découle de l'analyse structurelle et qui est définie non par le volume, mais plutôt par une "particulisation ", à partir d'une "particule " d'un matériau choisi. Dans son article et son introduction à la version anglaise des livres Small Architecture/Natural Architecture, Thomas Daniell résume en quelques mots la méthode de Kuma : "sa formule de conception est maintenant bien établie : sélectionner un seul matériau, le façonner en un petit module, puis le multiplier pour produire le bâtiment entier» (Daniell 2014 : 101).

Kuma applique sa méthode en dialoguant directement avec les artisans, en expérimentant la nature de chaque matériau, que ce soit le bois, le bambou, le papier ou la pierre. «Au lieu de l'ordre habituel des choses, [...] nous devrions faire des maquettes pour étudier des matériaux et des détails spécifiques dès le début » (Kuma 2015 : 21). Ce contact direct avec les artisans répond à l'intérêt de cet architecte pour les matériaux et l'innovation en matière de construction. Ce dialogue l'a ainsi invité à mettre de côté toute idée formaliste, en privilégiant les matériaux eux-mêmes - principalement naturels - afin de créer une architecture qui disparaît; ou, comme il la désigne, un anti-objet (Bognar \& Kuma 2005 : 14). Il précise que son architecture anti-objet ne nie pas la tangibilité et la présence des bâtiments, mais critique plutôt la lourde présence d'objets massifs généralement isolés de leur environnement et souvent destinés à se démarquer plutôt qu’à se fondre dans leur milieu.

17. Ces expressions proviennent des ouvrages écrits par Kuma, notamment Anti-Object (2005) et Studies in Organic (2009). 


\section{2. Une stratégie de conception pour les concours internationaux}

En 2001, Kuma Kengo a reçu en Italie le Prix international d'architecture en pierre, décerné pour la conception du musée de la Pierre de Tochigi au Japon (2000). Un second tournant s'est produit lorsqu'il a reçu l'année suivante le prix "Spirit of Nature Wood Architecture ${ }^{18}$ " en Finlande pour le projet du musée Andō Hiroshige, construit en bois, également à Tochigi (2000). L'obtention de ce prix a permis à l'agence de se faire connaître et reconnaître à l'échelle internationale, notamment en Europe. C'est à ce moment-là que l'agence a commencé à recevoir des invitations pour participer à des concours internationaux tels que ceux des Structures éphémères dans la ville d'Athènes (2002), des nouveaux locaux de la Banque centrale européenne à Francfort (2003) et du musée de l'Histoire des Juifs polonais à Varsovie (2005). Cependant, l'agence n'en a remporté aucun.

Au cours de la période 2008-2016, l'agence KKAA a modifié sa stratégie conceptuelle, passant d'un objectif d'effacement de l'architecture à une approche tentant d'être plus attractive pour s'adresser à un public international. Dans ses écrits, Kuma fait largement référence à cette période de développement qui a nécessité de nouvelles expérimentations et des recherches en rapport avec la réflexion soutenue par l'agence. Il explique cette évolution - en collaboration avec son équipe - après avoir perdu plusieurs concours internationaux :

J'ai commencé à douter que les bâtiments doivent réellement disparaître dans l'environnement et devenir complètement invisibles. Une chose qui a déclenché ce changement a été l'échec à plusieurs concours. [...] Nous avons calmement comparé le projet gagnant au nôtre [en nous référant à nos projets candidats pour le musée Nam June Paik en Corée (2003) et le musée de l'Histoire des juifs polonais à Varsovie (2005)], et il semblait que les commanditaires ne voulaient pas d'une architecture qui "disparaît ". Nous avons finalement compris leur évidente envie d'un morceau d'architecture qui fasse une forte impression, puisqu'ils dépensaient beaucoup d'argent pour le construire. (Bognar \& Kuma 2009 : 8)

18. Ce prix international, créé en 1999 par une association finlandaise parrainée par l'industrie du bois en Finlande, n'existe plus aujourd'hui. Kuma est le deuxième lauréat de ce prix, après l'Italien Renzo Piano (né en 1937) et avant le Suisse Peter Zumthor (né en 1943), deux architectes plus âgés bénéficiant déjà à l'époque d'une reconnaissance internationale. 
Il y eut une discussion au sein du bureau après les résultats, et l'opinion générale a exprimé le fait que nous ne pourrions jamais gagner un concours avec une approche se centrant sur l'effacement de l'architecture. (Kengo Kuma and Associates 2009 : 38)

\section{3. Jouer avec les règles françaises}

$\mathrm{Au}$ cours de cette étape, Kengo Kuma and Associates a créé Kuma \& Associates Europe, baptisée KAE. La société a choisi Paris comme lieu d'implantation pour trois raisons : tout d'abord, sa situation au centre de l'Europe de l'Ouest, permettant aux membres du personnel de se déplacer facilement vers d'autres pays tels que l'Italie, la Suisse, l'Angleterre ou l'Espagne. Le deuxième facteur est - comme nous l'avons vu précédemment lié à la réglementation en France qui oblige les institutions publiques à lancer des appels d'offres pour la conception de nouveaux projets d'architecture, des opportunités que guettent les agences indépendantes. Troisièmement, et c'est le plus important, Kuma a remporté les concours du Centre des arts et de la culture de la ville de Besançon et du Fonds régional d'art contemporain (FRAC) de Marseille en 2007 (fig. 05). Fort de ces succès et compte tenu de la reconnaissance internationale dont l'agence a bénéficié, KKAA a décidé d'établir ce bureau à Paris ${ }^{19}$, non seulement parce que le premier grand concours international qu'il a remporté était en France, mais aussi parce que le système français est le plus ouvert : "C'est très facile de participer à des concours en France, [dans] d'autres pays, ce n'est pas si facile ${ }^{20}$ ". De plus, afin de renforcer sa présence en France, Kuma - à la différence de la majorité des autres architectes étrangers exerçant dans ce pays - a reçu un diplôme français de l'École spéciale d'architecture (ESA) de Paris, lui permettant d'obtenir la qualification d'architecte ESA en France, valable pour s'inscrire à l'Ordre des architectes. Ainsi, il a pu jouer avec les règles françaises, ayant plus de poids dans la conception de ses projets sans la présence obligatoire d'un associé local. Son bureau à Paris n'est donc pas une succursale mais une entité totalement indépendante.

19. Extrait d'un entretien des auteurs avec un partenaire de KKAA, février 2017.

20. Toutes les citations de Kuma Kengo sont des transcriptions d'un entretien enregistré par les auteurs en mai 2017. 

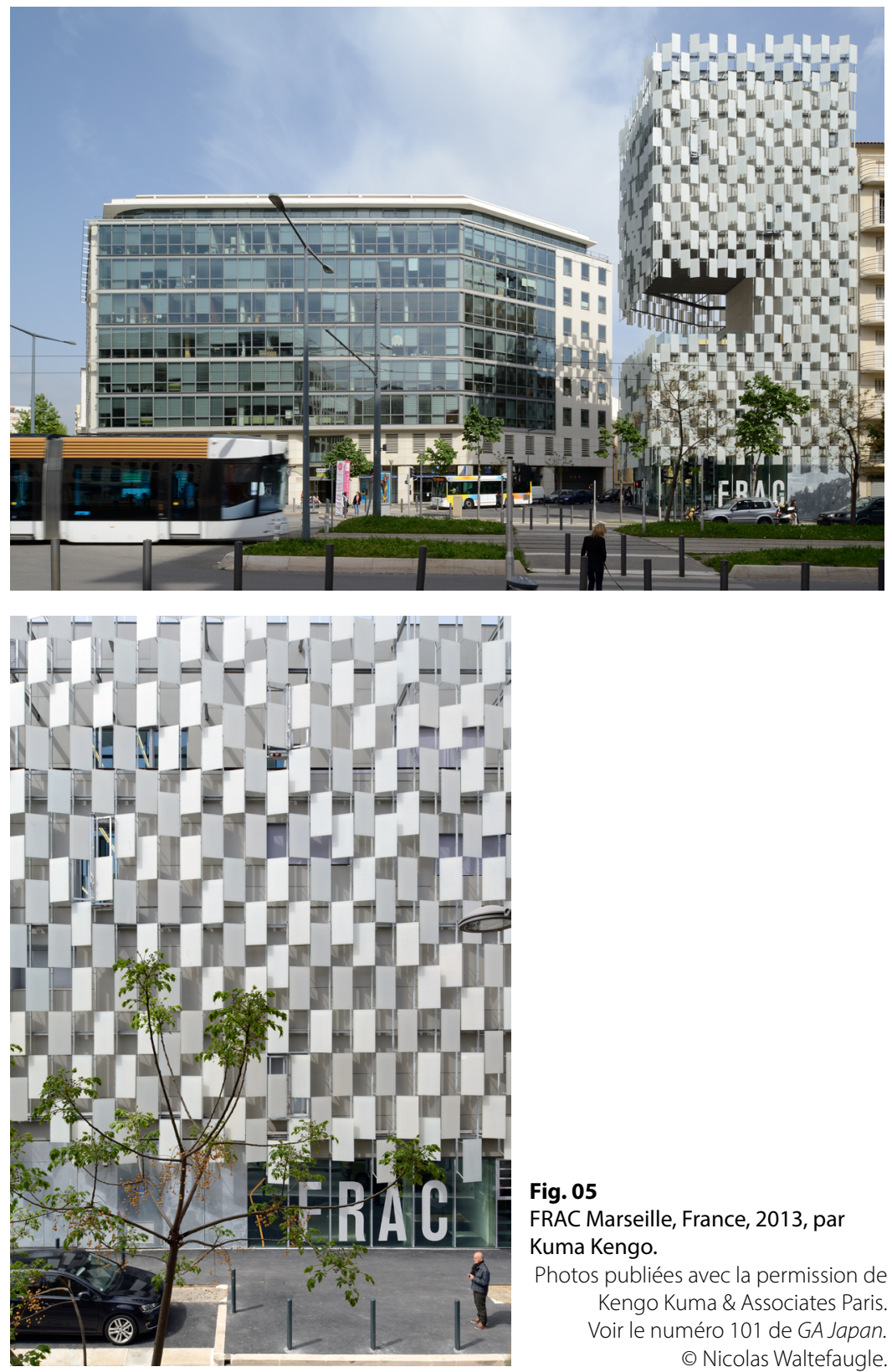

Fig. 05

FRAC Marseille, France, 2013, par Kuma Kengo.

Photos publiées avec la permission de Kengo Kuma \& Associates Paris. Voir le numéro 101 de GA Japan. (c) Nicolas Waltefaugle. 
Le cas de Kuma constitue donc un exemple de praticien qui fait le pont entre une méthode conceptuelle bien établie et une stratégie de gestion très claire. Le caractère local sur lequel il insiste par le biais du régionalisme et ce qu'il appelle l'" architecture organique ", sont mêlés à une pratique globalisée en expansion qui opère dans le monde entier. La création du bureau à Paris a permis à Kuma de "localiser" son architecture dans le contexte européen. Cette agence - après dix ans d'existence à Paris - est bien ancrée dans le milieu architectural français, en particulier après avoir tissé de solides relations professionnelles et techniques avec des ingénieurs et des entrepreneurs locaux.

L'un de ses derniers projets réalisés à Paris, les Archives d'Antoni Clavé (2017, fig. 06), révèle que Kuma est confiant dans la fusion de ses racines japonaises avec le contexte local. Malgré sa taille modeste, le projet manifeste la pensée de Kuma dans la pratique, à travers une collaboration avec des acteurs spécifiques: l'Atelier normand, une petite entreprise de construction implantée dans le Nord-Ouest de la France, qui réalise à l'origine des aménagements intérieurs de paquebots et diversifie son activité avec la construction de maisons individuelles; et Kobayashi Yasuo 小林康生, un fabricant de washi 和紙, papier japonais traditionnel, de Niigata. Kobayashi a fabriqué les écrans de washi dans l'Ouest de la France. L'Atelier normand, pour sa part, a fourni son expertise technique dans le domaine de la construction en bois pour la réalisation de l'intérieur du bâtiment ${ }^{21}$, révélant un échange enrichissant entre les savoir-faire des artisanats français et japonais. Kuma, - contrairement à Ban - utilise sa japonite ${ }^{22}$ comme un discours qui définit la matérialité de ses projets, et les processus à travers lesquels ils sont réalisés.

21. Extrait d'un entretien des auteurs avec l'ingénieur principal de l'Atelier normand concernant la collaboration avec le bureau de Kuma Kengo, juin 2017.

22. Japonité telle que l'a définie Isozaki Arata dans son ouvrage paru en 2003, Japanness in Architecture, où il présente un aperçu de ce qu'il identifie comme japonais dans les œuvres architecturales construites entre les $\mathrm{XVII}^{\mathrm{e}}$ et $\mathrm{XX}^{\mathrm{e}}$ siècles, en termes de goût, d'espace et de liens avec la nature. Il y fait référence aux caractéristiques propres du sanctuaire d'Ise, du temple Tōdai et de la villa impériale Katsura en particulier. 

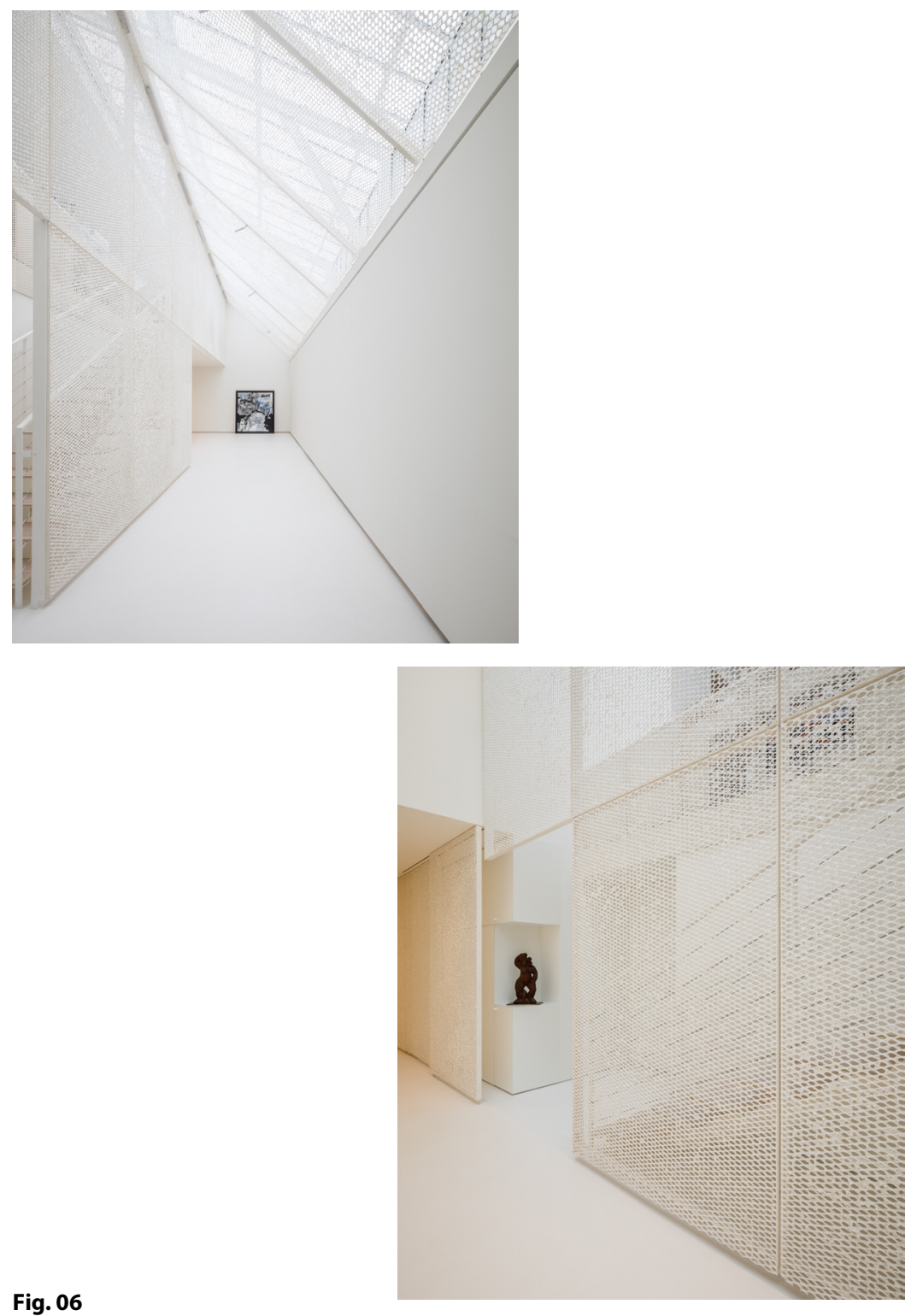

Fig. 06

Archives Antoni Clavé, Paris, 2017, par Kuma Kengo.

Photos publiées avec la permission de Kengo Kuma \& Associates Paris. (c) $11 \mathrm{~h} 45$. 


\section{Fujimoto Sou : échanges culturels et promotions mutuelles}

Fujimoto Sōsuke est l'un des architectes japonais les plus célèbres de sa génération qui, contrairement au système japonais de reconnaissance mutuelle entre maître et élève, a souvent été considéré comme un architecte rōnin 浪人, un samouraï sans maître.

Après ses études d'architecture à l'université de Tokyo, il suit le chemin de nombreux jeunes architectes japonais en s'appliquant à la conception de petits projets de maisons individuelles. Il crée son agence en 2000 à Tokyo et reçoit en 2005 pour son projet T-House une mention honorable au prix international de la revue britannique Architectural Review, dans la catégorie "Jeune architecture ». Il obtient également de cette revue, en 2006, le prix «Emerging Architecture » pour son projet de Centre psychiatrique pour enfants à Hokkaidō. En 2008, il gagne la médaille d'or de l'Institut japonais des architectes* et du Festival mondial d'architecture (WAF), dans la section "Maison individuelle ". " Ce prix a eu un grand impact sur moi ", dit Fujimoto, qui a grandi dans une petite ville au cœur de l'île de Hokkaidō avant d'entrer à l'université de Tokyo ${ }^{23}$. Grâce à ce prix, ajoute-til, il a été invité à participer à plusieurs projets nationaux et internationaux. "J'avais le temps, alors j'ai accepté toutes les invitations", se souvient-il. Ces voyages lui ont offert l'occasion de donner des conférences et de nouer des relations avec des confrères du monde entier. Ils ont en outre élargi ses perspectives : où qu'il atterrisse, il prend le temps d'explorer les lieux à pied, en se familiarisant avec l'architecture et l'organisation urbaine locales.

Ces expériences ont ouvert les yeux de Fujimoto sur la possibilité d'obtenir des commandes au-delà du Japon - une ambition qui continue à guider sa carrière aujourd'hui. La taille, la portée et le contexte des projets à l'étranger lui offrent des chances de conception qu'il n'a pas dans son propre pays (Pollock 2016). Ses réalisations locales telles Tokyo Apartment (2007), la Maison-N (2008, fig. 07) et la Maison-NA (2011, fig. 08), ont suscité l'intérêt des médias internationaux, ce qui a permis à Fujimoto d'asseoir sa carrière hors de l'Archipel : il a notamment été invité à concevoir

23. Extrait d'un entretien des auteurs avec Fujimoto Sou, avril 2017. 
le prestigieux pavillon Serpentine à Londres en $2013^{24}$. En 2008, il publie son manifeste Primitive Future, un livre bilingue qui a rencontré un grand succès, publié par INAX (aujourd'hui LIXIL), fabricant de sanitaires du Japon et dynamique promoteur de l'architecture et du design contemporains. Le travail de Fujimoto se fonde sur la recherche de nouvelles formes et interactions entre la nature et l'artifice. Son vocabulaire formel échappe à toutes les classifications aisées. Au cours de l'entretien, il nous explique que son concept de "futur primitif " se rapporte à la relation qui lie la " caverne ", c'est-à-dire l'habitation humaine primitive, à l'architecture du futur. Il cite les "grottes " et les «nids " comme les deux états embryonnaires de l'architecture. Pour lui, un nid est un endroit fonctionnel et organisé que les gens habitent confortablement; la grotte, par contre, est un espace "brut " que les gens doivent explorer pour trouver leur propre confort. La grotte est donc un espace accidentel que l'on est invité à utiliser de manière créative. C'est un espace, dit Fujimoto, qui se situe entre la nature et l'artefact, une forme informe.

24. Il s'agit d'un pavillon expérimental temporaire dont l'objet est de sensibiliser le public à l'espace architectural, commandé chaque année depuis 2000 à un architecte de renommée internationale par les Galeries Serpentine, un musée d'Art contemporain situé dans les jardins de Kensington. Les précédentes invitations ont notamment accueilli Zaha Hadid en 2000 et 2007, Itō Toyō et l'ingénieur Cecil Balmond (né en 1943) en 2002, Frank Gehry (né en 1929) en 2008, SANAA en 2009, les Ateliers Jean Nouvel en 2010, les suisses Herzog et De Meuron (nés en 1950) en 2012, la plupart "pritzkérisés ». 


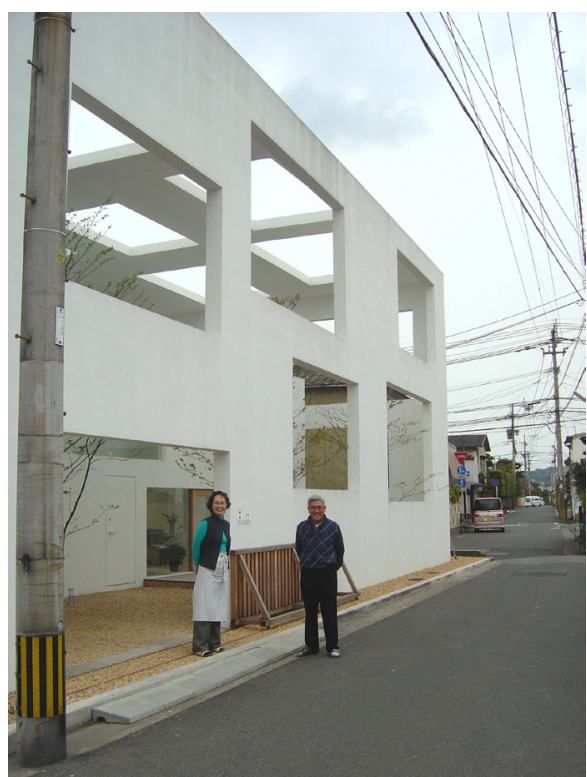

\section{Fig. 07}

Maison-N, Tokyo 2008 par Fujimoto Sou.

(c) Eduardo Pintos.

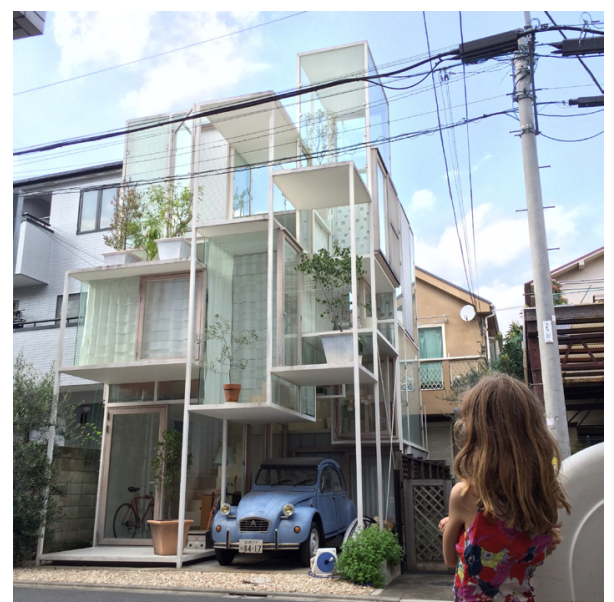

Fig. 08

Maison-NA, Tokyo 2011, par Fujimoto Sou.

C) Nicolás Boullosa. 


\section{1. Tokyo-Montpellier-Paris : un circuit inhabituel ou le détour par la collaboration franco-japonaise}

Sa relation avec la France a commencé lorsqu'il a été invité en 2014 par de jeunes architectes installés près de Paris à participer à un concours national d'architecture. "Les expériences de SANAA, Kuma Kengo, Ban Shigeru ou, avant eux, Itō Toyō et Andō Tadao encouragent les jeunes générations comme la mienne à vouloir faire des projets en France », précise Fujimoto (Albert \& Chessa 2016).

Nicolas Laisné (né en 1977) de l'agence Laisné-Roussel25 et Manal Rachdi (né en 1979) d'OXO Architectes l'ont approché dans l'espoir de collaborer avec lui pour concevoir un immeuble résidentiel à Montpellier, une invitation que Fujimoto a acceptée sans hésitation. «Fujimoto ne pouvait pas venir en France, alors nous avons fait le voyage au Japon et le projet est né là-bas, à Tokyo, lors d'un atelier de travail ", expliquent avec enthousiasme les deux architectes (Albert \& Chessa 2016). Le concours de Montpellier ${ }^{26}$ était unique en son genre en raison d'une part, de la spécificité du cahier des charges qui requérait une collaboration avec des architectes étrangers reconnus, et d'autre part, du programme qui demandait de concevoir une " folie ${ }^{27}$ ", un bâtiment capable de susciter l'intérêt et de changer la skyline de la ville.

Le projet dénommé "LAArbre blanc» (fig. 09), collaboration entre Fujimoto, Laisné-Roussel et Rachdi, a remporté le premier prix du concours. Par la suite, en moins de deux ans, entre 2013 et 2016, l'agence Sou Fujimoto Architects a remporté deux autres concours en France : un équipement d'enseignements mutualisés sur le campus de Paris-Saclay (en association encore avec Laisné-Roussel et Rachdi) en 2015, et un ensemble mixte de logements et bureaux près de la Porte Maillot - dénommé " Mille arbres » - sur le boulevard périphérique parisien (en association avec Rachdi), dans le cadre du premier appel à projet « Réinventer Paris » lancé par la municipalité en 2014. Marie de France, la directrice du bureau français de Fujimoto, rappelle que

25. Laisné travaille aujourd'hui indépendamment sous le nom "Nicolas Laisné Architectes ".

26. Un concours public de " conception-réalisation " lancé par la ville de Montpellier et intitulé "L'architecture du Xxi ${ }^{e}$ siècle : les folies architecturales".

27. Une folie désigne une maison de plaisance bâtie par les aristocrates ou les riches bourgeois de la ville et constitue une typologie de bâtiments caractéristiques de la périphérie au cours des XVIII ${ }^{\mathrm{e}}$ et XIX ${ }^{\mathrm{e}}$ siècles (Liotta 2019). 
"Fujimoto cherchait déjà à trouver le moyen d'ouvrir une agence en France ». Grâce à ces concours, sa renommée s'est peu à peu étendue et l'invitation à travailler sur le projet " Many Small Cubes » $(2014)^{28}$ - une petite installation temporaire dans le jardin des Tuileries - a permis de le faire connaître dans la presse généraliste, ce qui l'a aidé à renforcer sa présence médiatique en France. Comme à Londres, Fujimoto a tiré parti de la création d'une architecture temporaire qui raccourcit les temps de la réalisation et accélère la visibilité d'un savoir-faire.
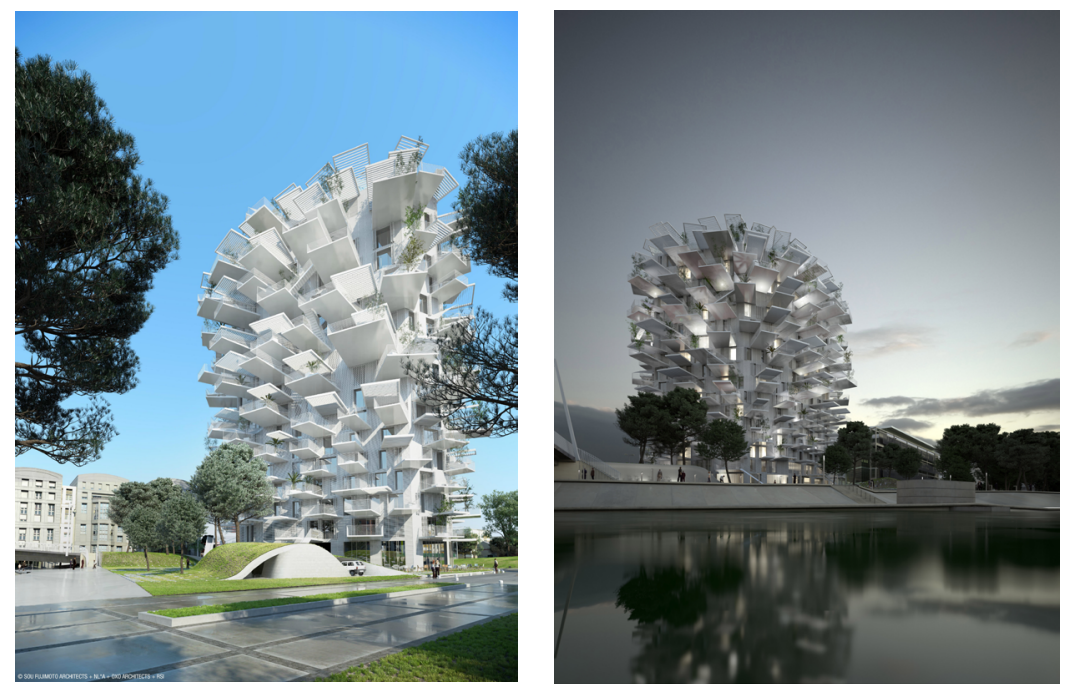

Fig. 09

L'Arbre blanc, Montpellier, 2019, par Fujimoto Sou.

Photos publiées avec la permission de Nicolas Laisné.

(c) Cyril Weiner.

28. Cette installation répond à un projet d'édition du galeriste Philippe Gravier, qui commande "de petites maisons nomades incarnant l'ambition de créer un nouveau concept entre art et architecture". Elle a été réalisée au sein de la Foire internationale d'art contemporain (FIAC), un an après le projet de Fujimoto pour les galeries Serpentine à Londres. 


\section{2. Une accélération de carrière grâce aux échanges culturels}

Larchitecte Manal Rachdi expose clairement les avantages de la collaboration avec Fujimoto: "Certains pensent que les architectes étrangers "volent" le travail des architectes français, mais en réalité ils permettent à de jeunes agences de travailler et d'accéder à des projets autrement inaccessibles ". Rachdi et ses confrères Nicolas Laisné et Dimitri Roussel ${ }^{29}$ (né en 1985) expriment leur joie en parlant de leur collaboration avec Fujimoto. Nicolas Laisné qualifie sa relation professionnelle avec Fujimoto d'équilibrée, ils échangent leurs idées de manière égale, libre et ouverte ${ }^{30}$ : "Lorsqu'un architecte étranger conçoit seul le projet et qu'un architecte local suit le chantier, c'est le pire des scénarios ", déclare Nicolas Laisné. "Nous comptons sur une collaboration de bout en bout. " De même, Fujimoto considère celle-ci comme une opportunité mutuelle. Il affirme : " [...] en collaborant avec eux, je peux élargir mon monde et eux le leur. L'important est de s'écouter les uns les autres, de s'inspirer".

Ainsi, et à l'instar de Ban, Fujimoto est arrivé à Paris après avoir remporté des concours d'architecture en France. Il s'est alors constitué un portfolio qui lui a permis d'étendre ses activités tant au Japon qu'en France, d'augmenter sa visibilité internationale et d'accélérer son parcours vers la renommée. Durant ces années, $90 \%$ de son travail au Japon porte sur des maisons individuelles, avec un seul projet de taille moyenne (moins de 3000 mètres carrés) : la bibliothèque de l'université d'art de Musashino (2010). Aujourd'hui, ses bureaux français et japonais comptent chacun plus de vingt employés.

29. Aujourd'hui à la tête de l'agence DREAM (Dimitri Roussel Ensemble Architecture Métropole).

30. D’après un entretien des auteurs avec Nicolas Laisné et Dimitri Roussel, février 2017. 


\section{Tane Tsuyoshi : un architecte japonais à Paris en pleine ascension au Japon}

Tane Tsuyoshi dirige actuellement son agence de Tokyo depuis son bureau parisien qui emploie près de trente personnes de diverses nationalités. Sa connexion avec la France s'est opérée de manière inattendue. Lors de sa deuxième année d'étude en architecture à l'université de Hokkaidō, il participe à un programme d'échange avec la Suède. Cela lui permet de visiter l'Europe pour la première fois, à l'âge de dix-neuf ans. Il se souvient de cette expérience : "Quand je pense aujourd'hui à cette période en Scandinavie, je me rends compte que ce fut un moment très important dans ma vie ${ }^{31}$ ".

Pendant son séjour d'une année en Suède, il visite également le Danemark. Là, il est fasciné par la culture et les modes de vie de la société scandinave. Au total, Tane Tsuyoshi passe un an en Suède et trois ans et demi au Danemark, entre études et travail. Puis, il décide de s'installer à Londres où il travaille pour un architecte renommé, David Adjaye (né en 1966). Tane considère cette période comme particulièrement productive et riche d'apprentissage grâce aux conseils d'Adjaye. C'est à cette époque qu'il rencontre ses premiers partenaires, Dan Dorell (né en 1973) et Lina Ghotmeh (née en 1980), qui travaillaient à Londres depuis un an sur un projet de Jean Nouvel. En 2006, en plus de leurs emplois réguliers, le trio décide de préparer le concours du musée national d'Estonie près de la ville de Tartu (fig. 10).

31. Toutes les citations de Tane Tsuyoshi sont extraites de la transcription d'un entretien mené en anglais par les auteurs en décembre 2018. 

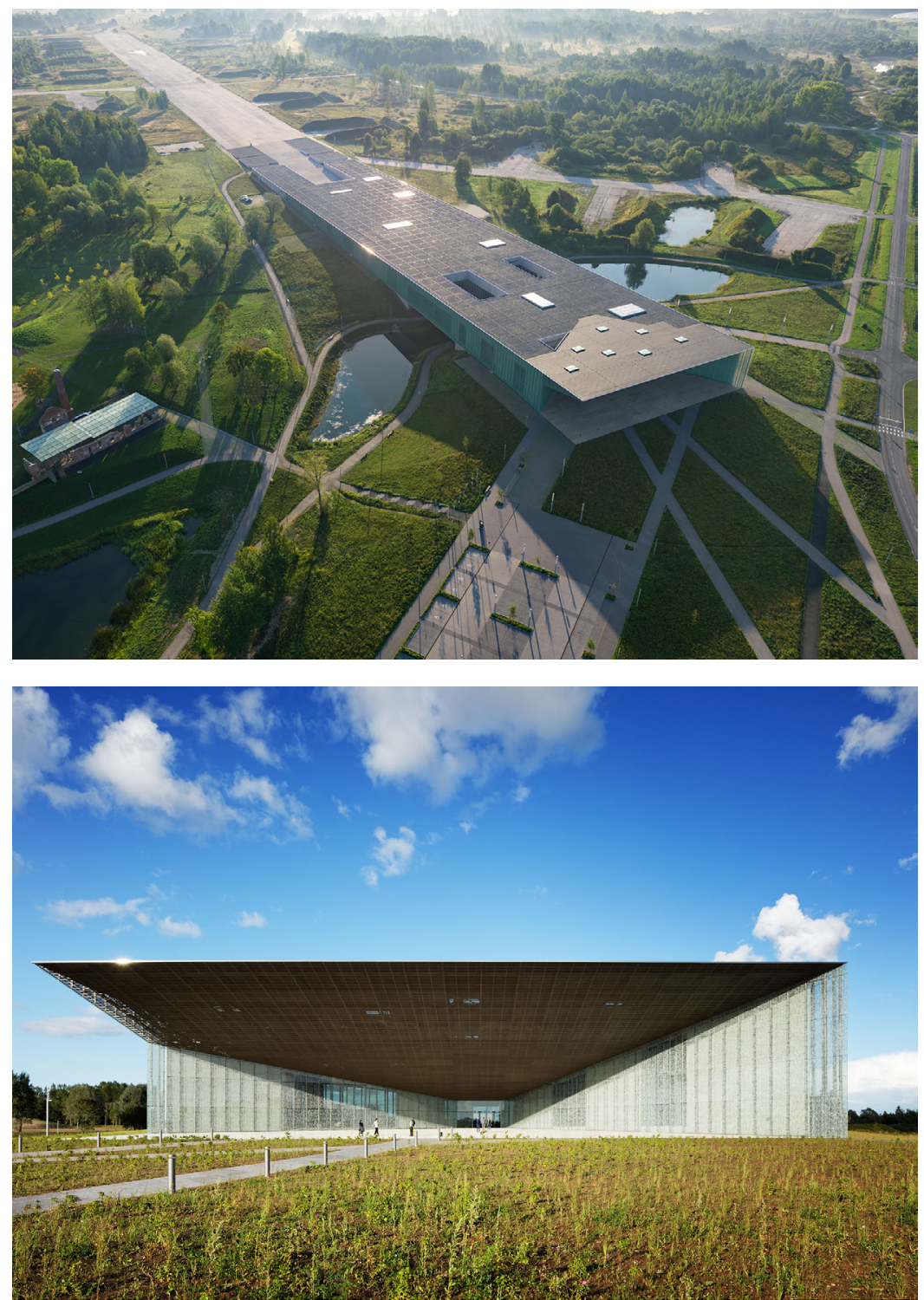

Fig. 10

Musée national d'Estonie, 2016, par DGT.

Photos publiées avec la permission de Tane Tsuyoshi. En haut : () Propapanda; en bas : @ Takuji Shimmura. 


\section{1. Un succès inattendu grâce à un concours international}

Tane précise que gagner ce concours était inimaginable. Les trois jeunes architectes abordent le projet à partir d'un point de vue extrêmement hardi car ils choisissent de ne pas se limiter au terrain officiellement assigné. Leur projet relie une ancienne piste d'atterrissage - une structure adjacente au terrain donné - au nouveau musée, et aborde ainsi de façon frontale un sujet de controverse pour le peuple estonien car l'histoire de cette infrastructure rappelle le lien douloureux avec la période soviétique; ce qui explique pourquoi les organisateurs du concours avaient renoncé à l'idée de l'intégrer. Tane affirme que son équipe n'ignorait pas les émotions suscitées par ce site dans la psyché nationale. Il pense qu’au contraire, leur équipe DGT - pour Dorell, Ghotmeh, Tane - a cherché à opérer une réappropriation de l'histoire en faisant de la piste une icône nationale pour l'avenir de l'Estonie. Après avoir reçu le premier prix, les trois architectes décident d'ouvrir un bureau à Paris, en s'appuyant sur l'expérience professionnelle de Dorell et Ghotmeh dans cette ville ${ }^{32}$. Tane précise :

Si nous l'avions ouvert à Londres, cela aurait été normal d'être une équipe internationale. Nous pensions qu'être présents à Paris en tant que Japonais, Libanaise et Israélo-Italien nous rendrait plutôt uniques dans le paysage architectural français. C'était stratégique, en un sens, pour nous démarquer.

Lors de sa création, l'équipe DGT n'avait qu'un seul projet : le musée national d'Estonie. Tirant parti du système français, elle participe à plusieurs concours publics, également au Japon et en Italie, mais n'en gagne aucun. Toutefois, cela lui permet d'obtenir une certaine visibilité médiatique, notamment lors du concours pour le nouveau stade olympique de Tokyo 2020 où son projet est classé parmi les dix premiers. Durant cette période, tout en enrichissant leur portfolio, les trois architectes acquièrent les rudiments de la gestion d'une agence, tels que le recrutement de personnels, la communication avec les clients et la collaboration avec les entreprises de construction.

32. Dorell a travaillé pour les Ateliers Jean Nouvel (AJN) à Paris entre 2004 et 2006; Ghotmeh a également travaillé pour AJN entre 2003 et 2006. 


\section{2. L'archéologie du futur en tant que pensée}

Créé en 2017, l'Atelier Tane Tsuyoshi Architects (ATTA) travaille actuellement sur de nombreux projets au Japon $-80 \%$ de son activité - et peu en Europe, une situation qui a conduit à multiplier les déplacements vers l'Archipel au cours de ces dernières années. En 2018, deux expositions à Tokyo (à la Galerie MA et à la Tokyo Opera City Art Gallery), consacrées aux projets de Tane, lui permettent de présenter son travail et sa pensée. En 2019, il est invité à la rencontre sur les musées du design au Xxi ${ }^{\mathrm{e}}$ siècle organisée à Tokyo par le Design Museum, une organisation liée au 21_21 DESIGN SIGHT fondé par l'architecte Andō Tadaō et le styliste de mode Miyake Issey 三宅一生 (né en 1938). Grâce à ces deux expositions et à cette invitation prestigieuse, Tane s'est reconnecté avec le Japon et s'intéresse - selon son point de vue - à ce qui s'y passe. Retombées de cette exposition médiatique, il a été invité à participer à de nombreux concours d'architecture, dont celui du musée de Hirosaki ${ }^{33}$ (inauguré en 2020, fig. 11) gagné contre Kuma Kengo. Tane tire profit de son expérience européenne par la mise en œuvre de la méthode qu'il a développée en travaillant sur le musée estonien, et qu'il appelle "recherche archéologique " ou " archéologie du futur ». Tane affirme que chaque lieu porte toujours une mémoire, profondément ancrée dans le sol :

Dans un premier temps, nous devons voyager et fouiller dans le temps, en creusant profondément comme des archéologues. Cette archéologie, le processus de réflexion en profondeur du passé vers le futur, devient lentement de l'architecture.

Tane croit fermement que les mémoires ne doivent pas appartenir au passé ou simplement servir de carburant à la nostalgie, mais qu'elles doivent plutôt devenir universelles : " [la mémoire] peut être une force motrice dans la création de l'architecture à venir ". Son processus de conception commence donc par une période de recherche intensive. Il fouille dans le passé pour trouver des traces mémorielles qui inspirent son processus de conception, comme en témoigne récemment le projet Todoroki House in Valley, une maison privée à Tokyo livrée en 2018.

33. D'après l'entretien avec Tane Tsuyoshi mené par les auteurs en décembre 2018. 

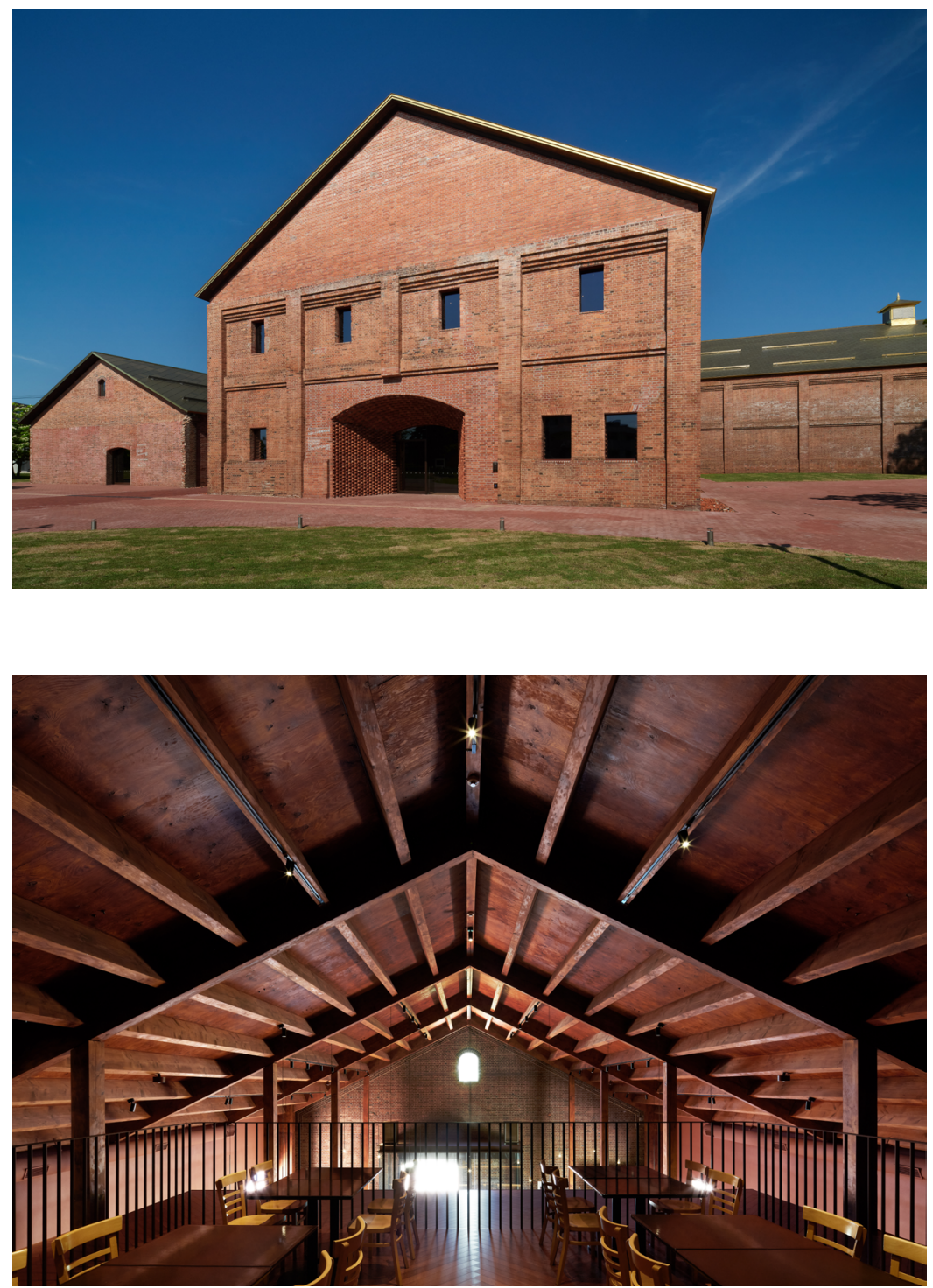

Fig. 11

Musée d'Art contemporain de Hirosaki, Japon, 2020, par Tane Tsuyoshi.

Photos publiées avec la permission de Tane Tsuyoshi.

(c) Daici Ano. 


\section{3. Liaisons entre Paris et Tokyo}

Tane se considère chanceux d'être à Paris, une ville où s'épanouissent de nombreux artistes japonais : entre autres des personnalités du théâtre, de la musique, de la mode. Sa présence en tant qu'architecte japonais à Paris le différencie et cette position particulière a incité plusieurs maîtres d'ouvrage à l'inviter à concevoir divers projets. De plus, les médias de l'Archipel ont commencé à s'intéresser au parcours, au travail et à la pensée de cet architecte japonais installé dans la capitale française.

Alors que Ban Shigeru, Fujimoto Sou et Kuma Kengo ont implanté un second bureau à Paris après avoir établi leur agence et leur pratique au Japon, le cas de Tane est clairement distinct. Il affirme :

Je n'ai pas suivi la carrière typique de beaucoup d'architectes japonais selon un apprentissage auprès d'un grand maitre pendant leurs années d'étude dans une université réputée. Je suis essentiellement allé de Tokyo à Hokkaidō et ensuite en Europe; il y a d'autres exemples similaires comme Andō Tadao ou Ban Shigeru et même Fujimoto Sou. Tous ont dû apprendre à partir de leurs expériences propres. Au cours de tels parcours, ces architectes ont développé leur approche spécifique et leur philosophie personnelle en amont.

Selon Tane, le plus grand risque qui menace aujourd'hui la profession d'architecte réside dans la préoccupation des concepteurs pour les " petits enjeux ». Il estime que le rôle de l'architecte est en train de s'affaiblir. Pour preuve, il prend l'exemple des entreprises de construction qui - au vu de leur taille et de leur pouvoir - ont de plus en plus de poids et de contrôle sur les projets, au détriment des jeunes architectes. Il ajoute :

Je sais la chance que j'ai eue d'être invité à des concours ou d'avoir des clients particuliers avec commande directe. Lorsque nous nous portons candidats à des concours, nous faisons attention à tous les paramètres. Nous avons par exemple remporté le projet du musée d'Art contemporain de Hirosaki, mais l'invitation à concourir n'a été adressée qu’à deux architectes, ce qui nous a donné davantage de chances de gagner. Je ne dirais pas que j'exploite le fait d'être un architecte japonais ou une marque japonaise comme stratégie pour obtenir de nouveaux projets.

Lors de notre entretien, Tane nous a confié qu'il était en train de préparer son entrée sur la scène architecturale en France. Il a une forte conscience du fait que sa visibilité est plus importante au Japon qu'en France et qu'il n'y connaît pas suffisamment les maîtres d'ouvrage ni les entreprises de 
construction. Il est en train de mieux comprendre comment monter un partenariat avec des architectes locaux et est conscient qu'il vit dans un pays où les architectes peuvent participer à des concours publics. Depuis 2017, il a ouvert un bureau de représentation au Japon, conservant son agence de Paris comme base principale pour le travail de conception. Le bureau de Tokyo sert de plateforme pour rencontrer les clients japonais et gérer les projets en cours. Il est important de mentionner qu'un tiers des membres du personnel sont des Japonais, capables de communiquer et de collaborer avec les clients, les entrepreneurs et les fournisseurs nippons.

\section{Le contexte architectural français : des opportunités aux défis pour les architectes japonais}

Les cas de Ban, Kuma, Fujimoto et Tane présentent chacun des particularités. À l'exception de Kuma, les trois autres figures en question n'ont pas enchaîné le parcours type des architectes japonais, celui du giri 義理, c'est-à-dire le protocole social traditionnel entre un maître et un apprentidisciple, qui est souvent resté d'usage malgré la modernisation entamée depuis l'ère Meiji (Steele 2017). Poursuivant cette tradition, Kuma a étudié à l'université de Tokyo sous la direction de Hara Hiroshi* 原広司 (né en 1936), architecte réputé, lui-même dans la lignée de Tange Kenzō. Ban, en revanche, a débuté son parcours aux États-Unis, Fujimoto à Hokkaidō puis à Londres et Tane en Europe; les trois ont donc tracé leur propre chemin sans suivre les pas d'un maître japonais.

Tane représente une exception puisqu'il a commencé son parcours depuis la France pour revenir au Japon; Kuma est le seul - parmi les quatre - à être habilité en France, il peut donc officiellement signer des projets en son nom propre; Fujimoto a été invité par des architectes français à collaborer avec lui avant d'ouvrir son propre bureau en France. Kuma et Ban ont apporté en France leurs conceptions innovantes des matériaux qui impliquent des technologies architectoniques mettant au défi le système de construction français. Chacun a choisi une approche particulière, ce qui prouve qu'il y a plusieurs voies possibles pour travailler en France en collaboration directe avec des associés locaux ou en employant du personnel français. Le contexte français offre à ces architectes, dotés de formations et de parcours différents, 
de nombreuses opportunités de collaboration et de création dans un environnement culturel riche.

La principale différence entre les contextes de la construction au Japon et en France, réside dans la nature collaborative de la culture des zenekon, absente en France. Ces dernières - comme nous l'avons déjà décrit - sont des entreprises de construction dont les valeurs sont issues d'une longue tradition de charpenterie. Le rôle du maître charpentier en tant qu'artisan à l'époque prémoderne était similaire à celui de l'architecte moderne qui conçoit et supervise tous les aspects de la construction du bâtiment : dépassant la conception, le charpentier était également un artisan qui combinait donc les deux fonctions de concepteur et de constructeur (Coaldrake 1990). Des architectes réputés, à la tête d'agences relativement modestes - atorie* アトリエ - ont mené à bien des projets complexes de grande envergure grâce à leur collaboration avec ces zenekon : la première phase de conception architecturale reste à un niveau schématique, laissant à l'entreprise la responsabilité des détails d'exécution et de l'ensemble de la construction. C'est le cas des réalisations complexes d'Itō Toyō, de Hasegawa Itsuko* 長谷川逸子 (née en 1941), de Kuma Kengo et d'autres (Buntrock 2002; Barr 2017).

Cette caractéristique constitue une différence majeure avec le contexte français où « les entreprises de construction veulent tout régler dès le début du processus ${ }^{34}$ ": le dossier d'appel d'offres puis les dessins d'exécution du projet, produits par les architectes, doivent être précisément détaillés, ce qui laisse très peu de marge pour d'éventuelles transformations au cours du chantier ${ }^{35}$, génératrices de surcoûts, de retards et de conflits. Cette

34. Les citations sont des transcriptions d'un entretien avec une cheffe de projet chez Kengo Kuma \& Associates (Paris) enregistré par les auteurs en juin 2017.

35. En France, un projet public comprend différentes phases d'études et de réalisations. La dernière phase d'étude s'appelle " études de projet " (PRO) qui a pour objet : "de préciser par des plans, coupes et élévations, les formes des différents éléments de la construction, la nature et les caractéristiques des matériaux et les conditions de leur mise en ouvre; d'établir un coût prévisionnel des travaux décomposés par corps d'état, sur la base d'un avant-métré; de permettre au maître de l'ouvrage, au regard de cette évaluation, d'arrêter le coût prévisionnel de la réalisation de l'ouvrage et, par ailleurs, d'estimer les coûts de son exploitation; de déterminer le délai global de réalisation de l'ouvrage " (Légifrance 2019). Par la suite, le dossier PRO est la base pour constituer un dossier, dit DCE (dossier de consultation entreprise), utilisé pour permettre aux entreprises de répondre. Une fois que le marché est attribué, les entreprises doivent réaliser 
dissemblance entre les deux pratiques a obligé ces architectes japonais à s'adapter afin de répondre aux contraintes de la construction en France. L'ancrage professionnel de Ban, Kuma et Fujimoto en France leur a permis d'intégrer leur mode de conception aux méthodes de construction et aux processus de coordination français. De plus, cette implantation leur a offert la possibilité d'élargir leur capacité en collaborant avec des intervenants locaux tels que des ingénieurs, des paysagistes et des experts en développement durable. La nature du processus de construction français a obligé ces architectes à consacrer plus de temps et d'énergie à détailler et déterminer leurs projets avant l'entrée dans la phase de construction. Quelques exemples significatifs du point de vue de leurs expériences de construction en France permettent de mesurer leurs différentes modalités d'adaptation au contexte local.

L'expérience de Ban en France l'a finalement amené à simplifier ses idées de conception et ses systèmes structurels. Le projet du Centre PompidouMetz a représenté un véritable défi pour les ingénieurs et les entreprises de construction français. Cela est dû à l'originalité structurelle de la toiture composée d'un treillis hexagonal de poutres en bois lamellé-collé, qui couvre le musée et enveloppe le bâtiment. Celui-ci est constitué de trois galeries rectangulaires en porte-à-faux qui traversent le bâtiment à différents niveaux et sont ancrées à un pilier principal en acier. La toiture a la forme d'un hexagone de 90 mètres de diamètre et d'une surface de 8500 mètres carrés. Sa structure en bois lamellé-collé est recouverte d'une membrane en fibre de verre blanche avec un revêtement de Téflon. Cette membrane, translucide la nuit et filtrant la lumière directe du soleil le jour, comprend un système autonettoyant.

Ban a conçu le projet du concours en collaboration avec Cecil Balmond (né en 1943), ingénieur renommé codirigeant à l'époque l'important bureau d'étude anglo-danois Ove Arup. Cependant, après la commande du projet, le travail de conception s'est poursuivi en collaboration avec une autre équipe d'ingénieurs en structure, du même bureau Ove Arup, et a finalement été achevé par l'ingénieur suisse Hermann Blumer en 2010.

des études d'exécution, le maître d'œuvre s'assure que les documents qu'elles ont établis respectent les dispositions du projet et, dans ce cas, leur délivre son visa. L'entreprise est responsable de la construction du projet (Légifrance 2019). 
Les poutres de la toiture ont été fabriquées à $95 \%$ en planches d'épicéa autrichien et suisse, le reste en hêtre français et en mélèze. La forme irrégulière de la toiture a nécessité la fabrication sur mesure de chaque poutre à l'aide de machines de découpe à commande numérique. Ces deux facteurs - l'importation de bois de l'étranger et la fabrication numérique de chaque pièce - ont lourdement pesé sur le budget et le calendrier du projet. Le coût initial de la construction s'élevait à 60 millions d'euros (Le Moniteur 2019), mais le coût final a atteint 69 millions d'euros, soit une augmentation de $15 \%$ (Colian 2019).

Dans deux précédents projets, le Hayek Center (Swatch Group) à Tokyo en 2007 et le country club Nine Bridges à Yeoju-Gun (2009) en Corée du Sud, Ban a développé pour les toitures un système structurel similaire à celui de Metz. En particulier, le toit du Centre Nicholas Hayek à Ginza présente une structure régulière, avec des éléments trois fois plus petits qu’à Metz, dont la réalisation a été entièrement pilotée par des ingénieurs et des entreprises de construction japonais. Leur mode opératoire a contribué à une livraison dans les délais et au respect du budget.

Pour sa deuxième œuvre majeure en France, la Seine Musicale à Boulogne-Billancourt (un centre culturel privé dédié à la musique, fig. 12), Ban a bénéficié des leçons tirées de l'expérience du Centre PompidouMetz. En effet, l'" œuf » - caractéristique majeure au centre du projet, qui abrite la scène principale - présente une forme régulière et plus simple. Tout d'abord, il ne s'agit pas d'une charpente en treillis, telle que celle qui a été utilisée dans des projets antérieurs. L'enveloppe de ce bâtiment est supportée par une structure en bois lamellé-collé de forme libre et géométriquement réticulée, dotée d'une voile photovoltaïque tournante. La structure s'étend sur 70 mètres dans l'axe longitudinal et 45 mètres dans l'axe transversal, avec une hauteur de 27,5 mètres. Elle est constituée de 1700 éléments en bois, uniques, assemblés pour créer ce réseau en bois lamellé-collé. Tous sont réalisés avec la même épaisseur, évitant ainsi la superposition complexe en trois couches de la structure du toit du Centre Pompidou-Metz. L'expérience de Metz a incité le bureau de Ban à Paris à repenser son processus de conception pour l'adapter aux compétences locales en matière de construction, aux matériaux, au coût et au calendrier. 

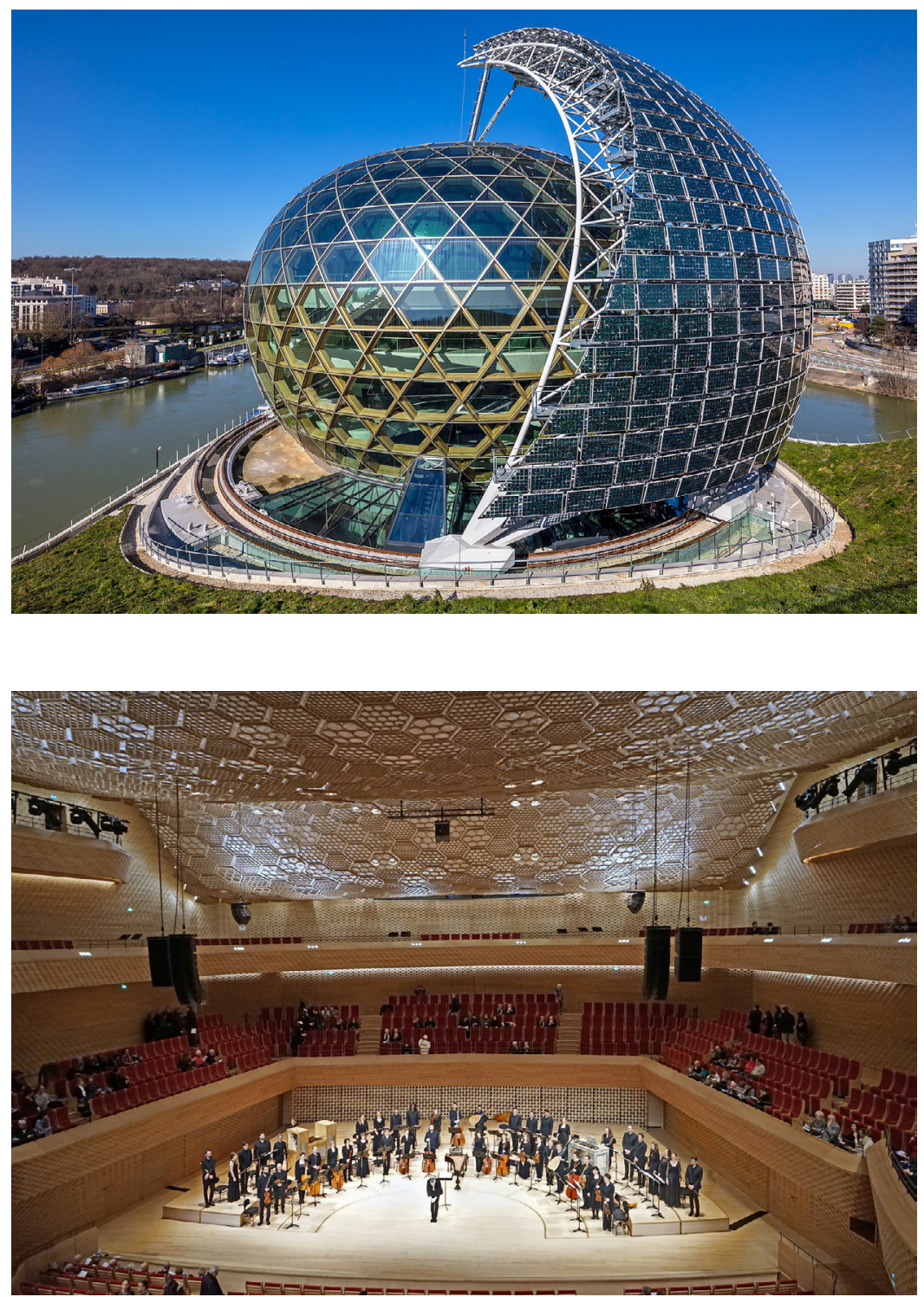

Fig. 12

La Seine musicale, Boulogne-Billancourt, 2017, par Ban Shigeru.

En haut: (๑) GraphyArchy. En bas : @ Jean-Pierre Dalbéra. 
Chez Ban, si c'est le Centre Pompidou-Metz qui l'a obligé à repenser sa façon de concevoir, chez Kuma, c'est le Forum culturel d'Aix-en-Provence, Conservatoire de musique et de danse, qui a joué ce rôle (fig. 13). Ce projet détient un record : pas moins de 10500 réserves ont été enregistrées dans le " procès-verbal de réception de l'immeuble " fin août 2013. Elles étaient relatives à des prestations "non exécutées " ou "non réalisées en fonction des règles de l'art ». La façade constituée de plaques d'aluminium pliées présentait des milliers de défauts. Conçue sur le principe de l'origami, elle couvre 8000 mètres carrés et comprend environ 1400 modules, dont la plupart ne sont pas standardisables. Lancé à l'été 2011 , le projet, d'un coût total de 16 millions d'euros, devait être achevé en septembre 2013. L'hôtel de Caumont - ancien siège du Conservatoire d'Aix-en-Provence - acquis par une fondation en 2012, devait être libéré pour des travaux de rénovation intérieure. Cette contrainte de calendrier a eu un impact majeur sur le sort du nouvel équipement. "Les délais étaient beaucoup plus courts que prévu et la mise en œuvre technique a pris beaucoup plus de temps que prévu ", explique Kuma. "La façade est d'une nature très différente de ce qui se fait habituellement en France. Au Japon, elle ne serait pas considérée comme particulièrement difficile " (Larrochelle 2014). Cette expérience stimulante a encouragé l'agence de Kuma à revoir aussi son processus de conception au Japon pour le faire mieux correspondre aux matériaux, aux techniques, aux coûts et aux délais stricts fixés en amont. 

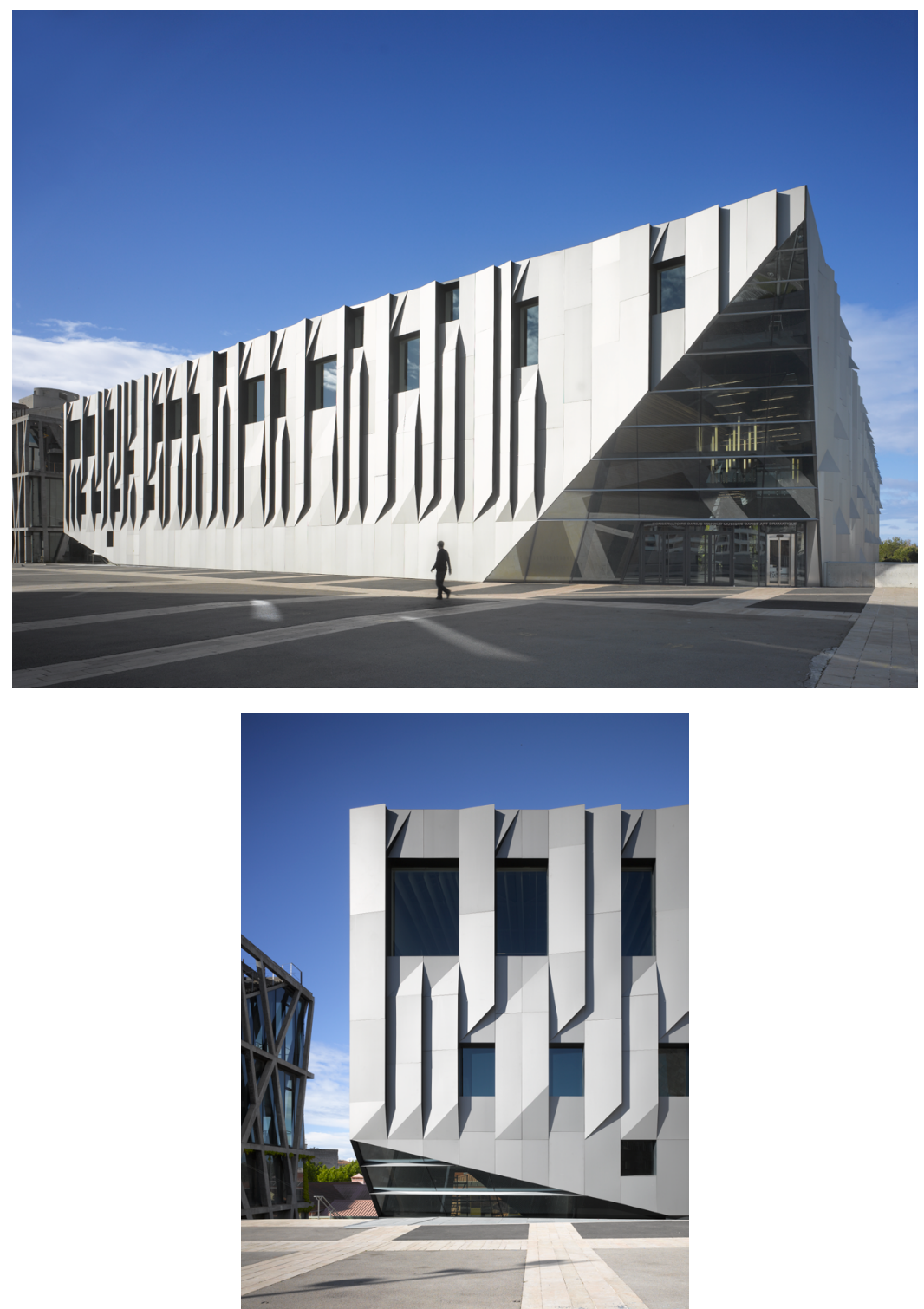

Fig. 13

Forum culturel d'Aix-en-Provence, 2013, par Kuma Kengo.

Photos publiées avec la permission de Kengo Kuma \& Associates Paris (c) Roland Halbe. 
Après cette expérience, les projets français conçus par Kuma \& Associates Europe (KAE) ont été élaborés avec des formes et des matériaux moins complexes, en particulier par rapport au concept de "molécularité » développé dans la réflexion de Kuma. Aujourd'hui, KAE a plusieurs projets majeurs en chantier en France : le musée Albert Kahn à Paris (2014-2021), le musée maritime de Saint-Malo (livraison prévue en 2022), le Centre de Congrès Sora dans l'Est de Paris (livraison prévue en 2023), le nouveau Parc des Expositions de Strasbourg (livraison prévue en 2022), l'immeuble One Hotel à Paris (livraison prévue en 2022), la gare Saint-Denis Pleyel (livraison prévue en 2030) - un bâtiment à usage mixte qui accueille un centre de congrès, des bureaux et des logements. En évaluant la date de livraison de chacun de ces projets, on peut supposer que KAE sera en activité en France pour au moins les dix prochaines années, voire au-delà. Après avoir tiré les leçons du projet d'Aix-en-Provence, KAE a dû et su s'adapter au contexte français, en appliquant des techniques constructives conformes aux capacités locales.

En revanche, Fujimoto n'a jusqu'à présent livré qu'un seul projet de grande envergure en France : l'immeuble résidentiel l'Arbre blanc à Montpellier. Conformément à sa philosophie de conception, sa stratégie constructive est essentiellement basée sur la simplicité. L'Arbre blanc est une tour au programme mixte: logements, équipements culturels et commerces sur 17 étages, d'une hauteur totale de 55 mètres et occupant une superficie de 10000 mètres carrés. Avec un noyau central construit selon une technique standardisée peu coûteuse - composé d'un bloc de services enchâssé dans une structure en béton armé -, l'Arbre blanc présente par contre une innovation au niveau de sa façade. Celle-ci comporte 193 balcons de formes différentes qui se ramifient et diversifient morphologiquement les appartements. Ces balcons terrasses - de 7 à 35 mètres carrés - sont conçus comme des espaces intermédiaires entre l'intérieur et l'extérieur, que les habitants peuvent utiliser de manières diverses. Leur disposition et leurs brise-soleil assurent une certaine intimité à chacun de ces espaces. Ils fonctionnent comme un dispositif spatial capable de redéfinir ce que pourrait être une tour méditerranéenne au XxI ${ }^{\mathrm{e}}$ siècle. Il ne s'agit plus d'un bâtiment fermé et vitré, habitable grâce à la climatisation, mais plutôt d'une architecture à la morphologie atypique et unique. L'ombre portée par les balcons et les brisesoleil contribue à protéger le bâtiment du soleil, réduisant ainsi de $30 \%$ les besoins de refroidissement et de climatisation. Leurs positionnements 
apparemment aléatoires permettent en outre d'optimiser les effets au vent typiquement produits par les immeubles de forme verticale.

L'Arbre blanc constitue un succès car il a atteint ses objectifs architecturaux, constructifs et financiers. Tous les appartements ont été vendus immédiatement, les résidents appréciant les terrasses comme un moyen de profiter de l'espace extérieur sous le climat méditerranéen. Malgré les apparences, l'immeuble a été facile à construire grâce à la simplicité de sa structure et à la modularité globale de la façade, sans compromettre sa qualité architecturale. Ce projet montre une collaboration réussie entre Fujimoto et ses partenaires français qui ont contribué, en employant leur expertise locale, à la réalisation d'un projet unique.

Tane, le plus jeune des quatre architectes présentés ici, n’a réalisé en France que de petits projets, tels que le pavillon temporaire Furoshiki ${ }^{36}$ (2018, fig. 14) construit devant l'hôtel de ville de Paris pour célébrer les 160 ans des relations diplomatiques entre la France et le Japon (Japonismes 2018), un espace de restauration au sein d'un entrepôt réhabilité à Paris, nommé Maison (2019, fig. 15), et la rénovation d'un appartement privé. L'agence travaille aussi sur un espace muséal de 400 mètres carrés dans l'hôtel de la Marine situé sur la place de la Concorde à Paris (Centre des monuments nationaux, livraison prévue en 2020). Il faut donc reconnaître que son parcours en France reste à s'accomplir avec la réalisation de projets plus conséquents.

36. Furoshiki 風呂敷 est une technique d'emballage traditionnelle japonaise dans un carré de tissu. 

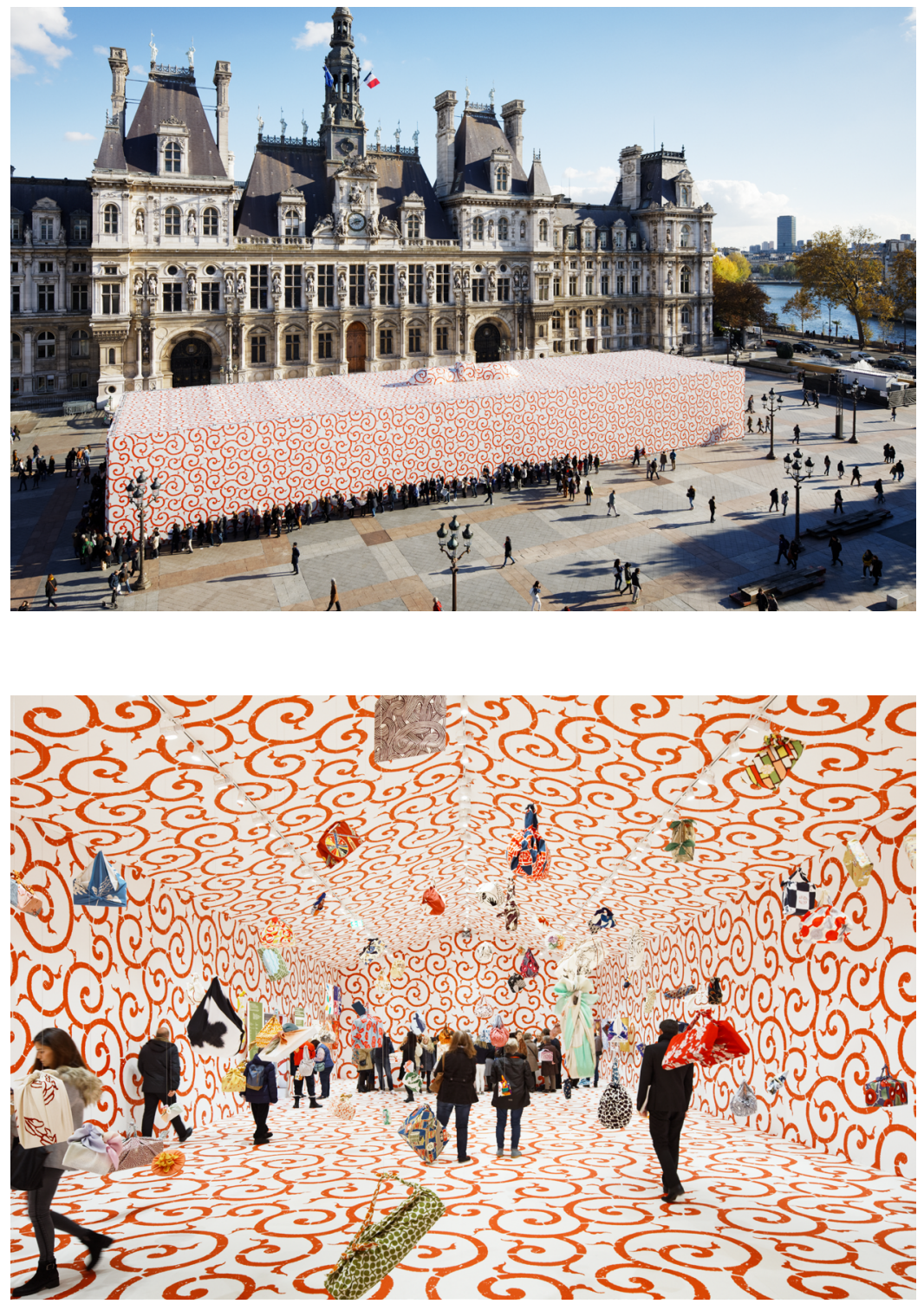

Fig. 14

Pavillon temporaire Furoshiki construit devant I'hôtel de ville de Paris, 2018, par Tane Tsuyoshi. Photos publiées avec la permission de Tane Tsuyoshi. (c) Takuji Shimmura. 

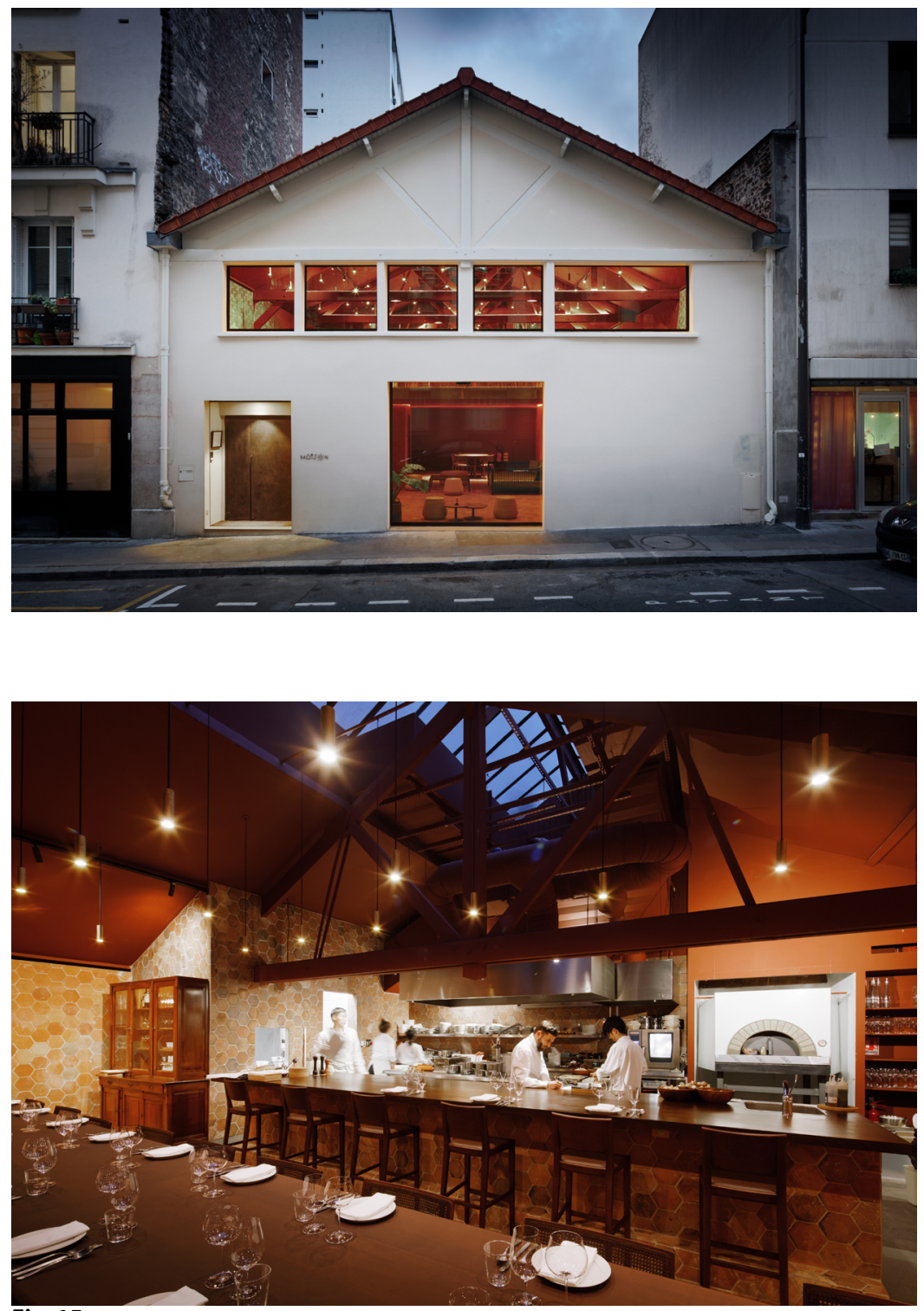

Fig. 15

Restaurant Maison, Paris, 2019 par Tane Tsuyoshi.

Photos publiées avec la permission de Tane Tsuyoshi.

(c) Takuji Shimmura. 
Le parcours de Tane renforce l'hypothèse d'Itō, à savoir que les jeunes architectes japonais ne sont pas intégrés dans leur société et trouvent en France un milieu où l'architecture a une valeur importante sur le plan social, culturel et urbain. Reconnu comme l'un des protagonistes de l'architecture japonaise en ce début de $\mathrm{XXI}^{\mathrm{e}}$ siècle, Tane a été propulsé dans le milieu japonais à travers et grâce à la France. Aujourd'hui, les projets en cours de réalisation au Japon témoignent de l'importance acquise par ce jeune architecte dans son pays natal : un stade de football à Tokyo (livraison prévue en 2027) situé à côté des stades de Tange Kenzō à Yoyogi, un musée d'Art contemporain à Teshima (livraison prévue en 2022) et un centre d'art

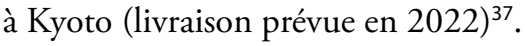

Il sera intéressant d'observer la carrière de Tane, l'évolution de son mode de conception et de sa stratégie professionnelle dans le contexte japonais, au cours des cinq prochaines années. Comment va-t-il s'adapter à la culture de la construction au Japon, après s'être formé en Europe pendant plus de dix ans? Comment son travail va-t-il évoluer par rapport à la culture spécifique des zenekon? Comment pourra-t-il gérer ses projets de plus en plus nombreux tout en restant implanté à Paris? Pour l'instant, l'agence a réorienté les profils de son équipe : si au tout début il avait surtout employé des architectes formés en Europe, dès qu'il a commencé à être sollicité pour des projets dans l'Archipel, Tane a dû s'entourer d'architectes japonais avec une expérience avérée au Japon.

\section{Conclusion}

La France demeure un pôle d'attraction de talents aux origines géographiques diverses, ce qui se traduit concrètement dans l'environnement bâti de ses villes. L'examen des pratiques de ces quatre architectes japonais permet de comprendre à la fois l'ouverture du marché français et la flexibilité de ces concepteurs. La question qui subsiste à ce stade est la suivante : leur présence en France est-elle en concurrence avec les architectes français ou sert-elle à mondialiser le milieu architectural français?

37. D'après l'entretien avec Tane Tsuyoshi mené par les auteurs en décembre 2018. 
L'implantation de ces quatre agences d'architecture au cours des deux dernières décennies s'inscrit dans la continuité d'un long échange établi entre les deux pays. Ce contexte a créé l'opportunité exceptionnelle pour de petites agences françaises - telles celles de Laisné-Roussel et Manal Rachdi - d'échanger leurs expérience et expertise avec des architectes japonais reconnus. Elle leur a également permis d'acquérir une reconnaissance internationale comme dans le cas de Laisné-Roussel qui a collaboré avec Fujimoto sur plusieurs projets, puis avec le bureau d'architectes SO-IL établi à New York.

Comme nous l'avons vu précédemment, le nombre d'architectes étrangers en France ne dépasse pas aujourd'hui 6,2 \% du nombre total des architectes inscrits à l'Ordre. Une autre donnée est à prendre en compte : en 2018, le chiffre d'affaires généré par les travaux du secteur de la construction publique s'élève à $21 \%$ du total du secteur de la construction en France (Ordre des architectes). Ces $21 \%$ sont répartis sur différents programmes de construction tels que le logement, les équipements culturels, les équipements éducatifs et autres. Comme les architectes étrangers ciblent principalement des projets publics et surtout culturels, on peut en conclure que la part des architectes étrangers - dont les Japonais - sur le marché français ne dépasse pas $5 \%$ du chiffre d'affaires total de la construction en France (Mutuelle des architectes français assurances $[\mathrm{MAF}]^{38}$; Leray 2019). Cette part mineure, mais hautement symbolique, éclaire bien les raisons qui incitent des architectes étrangers à s'investir en France : un marché ouvert et le prestige d'une architecture de haute tenue dotée de la valeur culturelle de bien public ${ }^{39}$. Les architectes français ne sont donc pas menacés par la présence d'architectes étrangers - et surtout pas par les architectes japonais; au contraire, cet échange enrichit le milieu culturel français et offre à des structures locales la possibilité de se développer au-delà des limites de leur pays. Réciproquement,

38. Les données et statistiques ont été communiquées par la MAF directement aux auteurs par courrier électronique en août 2019.

39. Le ministère de la Culture a lancé en 2019 une consultation sur les "valeurs de l'architecture " car elles sont bien mises à mal actuellement, et la profession est très inquiète de sa dégradation. 
l'implantation de ces quatre agences en France leur a permis d'élargir leur rayonnement international.

Un aspect à souligner est le caractère quasi artisanal des agences françaises, dont la grande majorité est de petite taille. Ce contexte a facilité l'adéquation et l'intégration des agences japonaises de même catégorie - telles que celles de Ban ou Fujimoto - dans le milieu architectural français. Ces architectes japonais produisent en France une sorte d'architecture de haute couture, même si leur structure professionnelle locale a atteint une taille comparable à celle des plus importants bureaux français. Ce n'est donc pas un hasard si les agences de grande taille sont quasiment inexistantes en France : les bureaux du type super zenekon ou américains préferent d'autres marchés qui correspondent à leur échelle, à leur système de fonctionnement et à une vision plus commerciale et moins culturelle de l'architecture.

Comme déjà mentionné, Ban, Fujimoto et Tane connaissent des parcours non conventionnels par rapport à la tradition japonaise, ce qui leur a permis de franchir les limites du Japon en se lançant dans une expérience internationale, non seulement en participant à des concours à l'étranger, mais aussi en s'installant dans un contexte totalement nouveau, au cœur de l'Europe. Il est important de noter que Ban a également fondé une agence à New York tandis que Kuma en a ouvert deux en Chine, l'une à Shanghai et l'autre à Pékin. Ainsi, même si le parcours de Kuma semblait traditionnel au début, l'évolution de son bureau prouve le contraire : outre son expansion internationale, il est le seul parmi les quatre à avoir engagé plus de 200 architectes, créant ainsi une grande entreprise d'architecture bien différente de la typologie courante des atorie.

$\mathrm{Si}$, pour Kuma, Ban et Fujimoto, travailler en France constitue une opportunité unique de développer leur activité hors du Japon, le cas de Tane se présente comme l'inverse car la France est son point de départ et le Japon est devenu son terrain d'expansion. Leur présence en France contribue à pallier la mise à l'écart des architectes du marché de la construction au Japon, leur permettant d'explorer une forme transnationale puis d'internationalisation à travers des projets en France et potentiellement dans d'autres pays d'Europe. En effet, leur installation à Paris a facilité leur implantation régionale, à proximité d'autres marchés européens. Par exemple, le bureau de Kuma à Paris est piloté comme une plateforme pour des projets en Italie (gare internationale de Susa), en Suisse (centre 
de l'École polytechnique de Lausanne) et au Royaume-Uni (Victoria and Albert Museum).

In fine, le contexte actuel de la commande architecturale publique et de l'exercice du métier d'architecte en France, rend possibles des échanges jugés mutuellement bénéfiques par et pour les acteurs impliqués. Pour les agences d'architecture japonaises, la France offre à l'architecte un rôle culturel reconnu par l'État et la société civile. Cependant, la situation va probablement évoluer dans un sens moins favorable pour les architectes et l'architecture, du fait de la remise en question - dans ce pays aussi - de l'architecture en tant que bien public ${ }^{40}$.

40. Ces dernières années, le domaine de l'architecture comme bien public est érodé par de nouveaux dispositifs législatifs. En particulier, la loi ELAN (2018) - portant sur l'évolution du logement, de l'aménagement et du numérique - autorise les organismes d'HLM, les SEM (sociétés d'économie mixte publiques locales) de construction et de gestion de logements sociaux, ainsi que les centres régionaux d'œuvres universitaires et scolaires (CROUS) à ne pas lancer de concours d'architecture. L'article 5-1 sur l'architecture du 3 janvier 1977 a été modifié en ce sens. Les maîtres d'ouvrage concernés demeureront toutefois soumis aux règles de publicité et de mise en concurrence. L'ordre national des architectes et plusieurs architectes ont protesté contre cette loi (Biau 2020). 


\section{Bibliographie}

\section{Albert Marie-Douce \&}

Chessa Milena 2016

«Ces architectes qui choisissent la

France », Le Moniteur, 26 février.

www.lemoniteur.fr/article/ces-architectes-

qui-choisissent-la-france.1071544

(dernière consultation le 6 mars 2019).

Architectural Institute of Japan 2019

«Architects and Building Engineers

related to AIJ », 14 avril.

www.aij.or.jp/eng/about/a_e.

html\#Architects\%20and\%20Engineers

(dernière consultation le 12 mars 2020).

\section{Assemble Media Group 2019}

"The World's Largest Architecture

Practices (WA 100) ».

https://cloud.3dissue.

com/176015/176406/205854/WA100/

index.html (dernière consultation le

16 janvier 2020).

BARR John 2017

«Zenecon: The Japanese Construction

Phenomenon », John Barr Architect.

www.johnbarrarchitect.com/single-

post/2017/05/01/Zenecon-The-Japanese-

Construction-Phenomenon (dernière

consultation le 3 mars 2019).

BIAU Véronique 2020

Les Architectes au défi de la ville néolibérale, Marseille, éditions

Parenthèses.
Bognar Botond, Frampton Kenneth \&

OtA Kayoko 2000

Nikken sekkei: Building Future

Japan 1900-2000, New York, Rizzoli

International Publications Inc.

Bonnin Philippe, NisHida Masatsugu \& InAGA Shigemi (dir.) 2014

Vocabulaire de la Spatialité Japonaise, Paris, éditions du CNRS.

\section{Buntrock Dana 2002}

Japanese Architecture as a Collaborative Process: Opportunities in a Flexible Construction Culture, New York, NY, Routledge-Taylor \& Francis Group.

Centre de coopération industrielle UEJapon 2015

"Sustainable building and construction sector in Japan and analysis of opportunities for European firms » www.eu-japan.eu/sites/default/files/ publications/docs/sustainableconstruction final.pdf (dernière consultation le 2 septembre 2020)

\section{Centre des monuments nationaux 2019}

« La AL Thani Collection Fondation a choisi Atelier Tsuyoshi Tane Architects pour la conception de son nouvel espace muséal à l'Hôtel de la Marine », 9 août.

https://presse.monuments-nationaux.

$\mathrm{fr} /$ Espace-presse/Repertoire-presse/

La-Al-Thani-Collection-Fondation-a-

choisi-Atelier-Tsuyoshi-Tane-Architects-

pour-la-conception-de-son-nouvel-

espace-museal-a-l-Hotel-de-la-Marine. 
-Ouverture-prevue-au-printemps-2020

(dernière consultation le 19 mai 2020).

\section{Coaldrake William 1990}

The Way of the Carpenter: Tools and Japanese Architecture, New York, Weatherhill Inc.

Daniell Thomas \& Birnbaum Alfred 2014 «Acting Natural », AA Files, 69 : 100-106.

\section{Frampton Kenneth 2013}

Kengo Kuma: Complete Works, Londres, Thames \& Hudson.

\section{Frampton Kenneth \& Kudo Kunio 1997} Japanese Building Practice: From Ancient Times to the Meiji Period, New York, Van Nostrand Reinhold.

\section{Fuлммото Sou 2008}

Primitive Future, Tokyo, INAX Publishing.

Fuлıмото Sou 2015

Architecture Works 1995-2015, Tokyo, TOTO Publishing.

GıUstı Julien 2017

"Pourquoi une loi sur l'architecture? ", Fédération nationale des Conseils d'architecture, d'urbanisme et de l'environnement.

www.fncaue.com/pourquoi-une-loi-surlarchitecture/ (dernière consultation le 30 mai 2020).

\section{IsOZAKI Arata 1978}

MA: Espace-temps du Japon (catalogue du festival d'automne au musée des Arts décoratifs, Paris).

\section{Isozakı Arata 2003}

Japan-ness in Architecture, Cambridge, Massachusetts, The MIT Press.

ITô Toyô 2014

L'Architecture du jour d'après, Paris, Impressions nouvelles.

JACQUet Benoît, Matsuzakı Teruaki \& TARDITs Manuel 2019

Le charpentier et l'architecte: une histoire de la construction en bois au Japon, Lausanne, Presses polytechniques et universitaires romandes.

JodıDıo Philip 2015

Shigeru Ban: Complete Works 1985-2015, New York, Taschen America Llc.

\section{JodıDıo Philip 2016}

Shigeru Ban: Architecture of Surprise, New York, Taschen America Llc.

\section{Journal Officiel de la République} française 2017

$J O R F, 50$, Service public de la diffusion du droit.

www.legifrance.gouv.fr/affichJO. do?idJO=JORFCONT000034096714

(dernière consultation le 5 mars 2019).

\section{Journal Officiel de la République} française 2018

Loi $n^{\circ} 77-2$ du 3 janvier 1977 sur I'architecture, Service Public de la Diffusion du Droit. www.legifrance.gouv.fr/affichTexte.do?cid Texte=JORFTEXT000000522423 (dernière consultation le 5 avril 2019). 
Kofler Andreas 2017

Architectures japonaises à Paris, 18672017, Paris, Pavillon de l'Arsenal.

\section{Kuma Kengo 2008}

The Anti-Object, Londres, Architectural Association.

\section{Kuma Kengo 2009}

Studies in Organic, Tokyo, Toto.

\section{Lago-Novás Domingo Juan 2014}

« Diseñando [el estudio de] arquitectura: modelos de negocio exitosos » (Concevoir [l'atelier d'] architecture : des modèles commerciaux à succès), thèse de doctorat à I'Universidad Europea Madrid, Espagne.

\section{LARRochelle Jean-Jacques 2014}

"Les mauvais plis du conservatoire d'Aix-en-Provence », Le Monde, 3 janvier. www.lemonde.fr/culture/

article/2014/01/03/les-mauvais-

plis-du-conservatoire-d-aix-en-

provence_4342511_3246.html (dernière consultation le 16 avril 2019).

\section{Légifrance}

«Missions de maîtrise d'œuvre confiées par des maîtres d'ouvrage publics à des prestataires de droit privé » www.legifrance.gouv.fr/affichTexte.do?ci dTexte $=$ LEGITEXT000006067890\&dateTe $x t e=20200706$ (dernière consultation le 6 juillet 2020).

\section{LemoIne Bertrand 2019}

«Architectes étrangers en France : une stratégie? ", Archistorm, 96, mai-juin : 98-102.

\section{LeRAY Christophe 2019}

«France terre d'accueil », Chroniques

d'architecture, 22 janvier.

https://chroniques-architecture.com/

france-terre-daccueil/ (dernière

consultation le 30 août 2019).

LıOTTA Salvator-John \& LouYot Fabienne

2017a

«Designing Social System: the

Architecture of Laisné-Roussel »,

Domusweb, 14 février.

www.domusweb.it/en/

architecture/2017/02/14/laisne_

roussel.html (dernière consultation le

12 mars 2020).

LıOTTA Salvator-John \& LouYot Fabienne 2017b

"Shigeru Ban: Serving Society with

Architecture », Domusweb, 5 octobre.

www.domusweb.it/en/

architecture/2017/10/05/shigeru-ban-

-la-mia-architettura-al-servizio-della-

societa.html (dernière consultation le

12 mars 2020).

\section{LıOTTA Salvator-John 2019a}

"Kengo Kuma and Associates », 100+Best Architecture Firms, supplément spécial à Domus, 1033, mars : 51-52.

\section{LıOTTA Salvator-John 2019b}

« White Tree », Domus, juillet-août 2019, 1037 : 766-773.

Mirza \& Nacey Research 2018

« La Profession d'architecte en Europe 2018 », Bruxelles, Conseil des architectes d'Europe. 
www.ace-cae.eu/fileadmin/

New_Upload/7._Publications/

Sector_Study/2018/2018_ACE_Report_

EN_FN_5.pdf (dernière consultation le

18 janvier 2019).

\section{Nikken sekkei 2019}

« About: Corporate Data »

www.nikken.co.jp/en/about/outline.html

(dernière consultation le

20 janvier 2019).

Nogue Nicolas 2010

"Les Architectes étrangers en France", étude pour le Conseil national de l'Ordre des architectes.

www.architectes.org/sites/default/files/

atoms/files/les-architectes-etrangers-

en-france.pdf (dernière consultation le

22 août 2019).

NuIJsink Kathelijne 2012

How to Make a Japanese House,

Rotterdam, Nai Publisher.

Ordre des Architectes, France, 2018

Archigraphie 2018: Observatoire de la profession d'architecte, Paris, CRÉDOC, Centre de recherche pour l'étude et l'observation des conditions de vie. www.architectes.org/sites/default/files/ atoms/files/cdp-64-web.pdf (dernière consultation le 22 décembre 2020).

Pollock Naomi 2016

"Sou Fujimoto: "Foreigners Don't Want

Really Crazy Things"», The Architectural

Review, 24 octobre.

www.architectural-review.com/essays/

profiles-and-interviews/sou-fujimoto-

foreigners-dont-want-really-crazy- things/10014022.article (dernière consultation le 5 avril 2019).

ReYNolds Jonathan 2004

«The Formation of a Japanese

Architectural Profession ", in Takeuchi

Melinda, The Artist as a Professional in

Japan, Stanford, Stanford University

Press : 180-236.

ShiкATA Yutaka \& Abe Takumi 2017

KPF Kohn Pedersen Fox Innovation,

Architecture and Urbanism, Tokyo,

Yoshida Editions.

Steele James 2017

Contemporary Japanese Architecture:

Tracing the Next Generation, New York, Routledge.

TANE Tsuyoshi 2018

Archaeology of the Future, Tokyo, TOTO Publishing.

\section{The Japan Institute of Architects} 2014-2015

« Country Report », Tokyo, The Japan Institute of Architects. 


\section{ANNEXE}

\section{Méthodologie de recherche}

Cette étude est basée sur les statistiques établies par le Conseil national de l'Ordre des architectes (CNOA) en France. Elle s'inscrit dans le cadre d'une recherche plus vaste qui examine les données relatives à la profession d'architecte et au marché français de la construction.

En plus des données statistiques, notre méthodologie pour cette étude s'est également appuyée sur des rencontres avec les quatre architectes japonais ayant pour point commun d'avoir implanté un bureau en France.

Les entrevues mentionnées dans le présent document ont été menées par les auteurs au cours de différentes périodes entre février 2017 et décembre 2018. Des entretiens directs en face à face avec Ban Shigeru, Kuma Kengo, Fujimoto Sou et Tane Tsuyoshi ont été effectués. En outre, les auteurs ont également mené d'autres entretiens formels et des discussions informelles avec des membres du personnel travaillant dans les bureaux de Kuma Kengo à Tokyo et à Paris dans le cadre d'un travail de terrain effectué pour une recherche plus large sur la pratique du design de Kengo Kuma \& Associates.

Les entretiens ont été enregistrés, transcrits et utilisés pour plusieurs parties de l'article. Ils ont été conduits soit en anglais, soit en japonais, selon la préférence de l'architecte interrogé. Leurs durées variaient entre un minimum de 30 minutes et un maximum de 90 minutes.

Le format de l'entrevue diffère d'un architecte à l'autre, en fonction des intérêts architecturaux et du processus de conception de ce dernier. Cependant, tous les entretiens ont abordé des questions en rapport avec les conditions qui ont motivé chaque architecte à ouvrir son cabinet à Paris, les différences entre le Japon et la France en termes de processus de construction. De plus, les auteurs se sont concentrés sur les stratégies de conception et de gestion utilisées pour mener à bien leurs projets en France - et en Europe.

Les questions étaient ouvertes afin d'assurer la réception d'informations approfondies, en particulier les opinions, les comportements, les expériences des quatre architectes interrogés. Ces discussions ont 


\section{ANNEXE}

fourni des données qualitatives à partir desquelles les auteurs ont pu tirer des conclusions. Il est important de mentionner que - même si l'objectif de ces entretiens était d'extraire des données qualitatives les auteurs ont récupéré des données quantitatives indicatives lors de leur visite dans le bureau des architectes, qui comprennent le nombre d'employés dans chaque bureau, le nombre de projets, le ratio entre le personnel d'architecture et le personnel administratif, etc.

Les entretiens réalisés s'inscrivent dans le cadre d'une recherche approfondie sur le processus de conception au sein des différents bureaux d'architectes japonais. Une première série d'entretiens avec Ban, Kuma et Fujimoto a été enregistrée entre mai et août 2017, tandis que l'entretien avec Tane a été réalisé en décembre 2018.

En particulier, la première série d'entretiens a été réalisé sous forme d'entretiens vidéo pour l'exposition intitulée: "What is Co-Dividuality? Architettura giapponese e la shared house di Farm Cultural Park ", organisée par Salvator-John A. Liotta et Fabienne Louyot pour la galerie d'art Farm Cultural Park à Favara, en Italie, qui s'est déroulée entre juillet 2017 et juin 2018.

Un deuxième entretien a été mené avec Ban en février 2018 dans le but de clarifier son choix de s'associer avec un architecte français local. Le dernier entretien a été réalisé en décembre 2018 avec Tane Tsuyoshi. L'idée d'organiser une rencontre avec Tane est apparue au fur et à mesure que les auteurs orientaient leur intérêt vers la montée en puissance des architectes japonais travaillant spécifiquement en France, et que les questions sur l'intérêt des architectes japonais pour la France devenaient plus éminentes. En outre, une courte rencontre a été organisée avec Fujimoto Sou en juin 2019 pour s'enquérir des informations supplémentaires relatives à son association avec les architectes français et des circonstances qui ont conduit à l'ouverture de son bureau à Paris.

En parallèle et dans le cadre de sa recherche doctorale à l'université de Tokyo, Aya Jazaierly a mené vingt-quatre entretiens formels avec des membres du personnel de Kengo Kuma \& Associates, et huit avec des collaborateurs qui travaillent pour KKAA tels que des 


\section{ANNEXE}

entrepreneurs, des clients et des ingénieurs. Cette recherche, intitulée " Kengo Kuma \& Associates between a Design Studio and an Architectural Enterprise ", porte sur les processus de conception et les procédures de gestion conçus dans le contexte de Kengo Kuma \& Associates (KKAA). Cette étude visait à rechercher, examiner et identifier les divers éléments et méthodes qui ont contribué - et continuent de contribuer - à la croissance de KKAA au cours des trois dernières décennies. Elle visait à positionner KKAA par rapport au développement de la pratique architecturale, en identifiant le modèle dans lequel il opère, en référence aux pratiques historiques et contemporaines à l'intérieur et à l'extérieur du Japon. Le travail de terrain consistait à observer quotidiennement les réunions, les modélisations, les discussions et les procédures se déroulant dans les bureaux de KKAA à Tokyo et à Paris. Il est important de mentionner que l'auteur a également mené des entretiens directs avec des architectes japonais de renom : Yamamoto Riken, Nishizawa Ryūe* 西沢立衛 (né en 1966), Aoki Jun 青木淳 (né en 1956) et Suzuki Edward [Edowādo] 鈴木エドワード (1947-2019) au cours des mois de juillet et août 2017.

\section{Liste non exhaustives des entretiens :}

\begin{tabular}{|l|l|l|l|}
\hline Entretien avec & Effectué par & \multicolumn{1}{|c|}{ Date } & \multicolumn{1}{c|}{ Lieu } \\
\hline Ban Shigeru & $\begin{array}{l}\text { Salvator-John } \\
\text { A. Liotta }\end{array}$ & 15 juin 2017 & $\begin{array}{l}\text { Agence Shigeru Ban } \\
\text { Paris, France }\end{array}$ \\
\hline Fujimoto Sou & $\begin{array}{l}\text { Salvator-John } \\
\text { A. Liotta }\end{array}$ & 21 juin 2017 & $\begin{array}{l}\text { Agence Sou Fujimoto } \\
\text { Paris, France }\end{array}$ \\
\hline Kuma Kengo & $\begin{array}{l}\text { Aya Jazaierly, } \\
\text { Salvator-John } \\
\text { A. Liotta }\end{array}$ & 28 juin 2017 & $\begin{array}{l}\text { Agence Kengo Kuma \& } 2017, \\
\text { Tokyo, Japon }\end{array}$ \\
\hline $\begin{array}{l}\text { Directrice de } \\
\text { projet chez Kengo } \\
\text { Kuma \& Asso- } \\
\text { ciates Europe }\end{array}$ & Aya Jazaierly & 20 juin 2017 & $\begin{array}{l}\text { Agence Kengo Kuma \& } \\
\text { Associates } \\
\text { Paris, France }\end{array}$ \\
\hline
\end{tabular}




\section{ANNEXE}

\begin{tabular}{|l|l|l|l|}
\hline \multicolumn{1}{|c|}{ Entretien avec } & Effectué par & \multicolumn{1}{|c|}{ Date } & \multicolumn{1}{c|}{ Lieu } \\
\hline $\begin{array}{l}\text { Associée et vice- } \\
\text { présidente chez } \\
\text { Kengo Kuma \& } \\
\text { Associates }\end{array}$ & Aya Jazaierly & 11 juillet 2017 & $\begin{array}{l}\text { Agence Kengo Kuma \& } \\
\text { Associates } \\
\text { Tokyo, Japon }\end{array}$ \\
\hline Ban Shigeru & $\begin{array}{l}\text { Salvator-John } \\
\text { A. Liotta }\end{array}$ & 10 février 2018 & $\begin{array}{l}\text { Agence Shigeru Ban } \\
\text { Paris, France }\end{array}$ \\
\hline Tane Tsuyoshi & $\begin{array}{l}\text { Salvator-John } \\
\text { A. Liotta }\end{array}$ & 8 décembre 2018 & $\begin{array}{l}\text { Agence Tsuyoshi Tane } \\
\text { Paris, France }\end{array}$ \\
\hline
\end{tabular}

\section{Sélection des principales questions soulevées lors des} entretiens :

- Pratique internationale : Comment avez-vous décidé d'ouvrir un bureau en France et pourquoi?

- Sur la base de votre expérience de travail dans plusieurs pays, quelles sont les différences entre la France et d'autres contextes? Nous nous référons ici au code de la construction, aux systèmes de concurrence, aux attentes des clients, aux matériaux, aux systèmes de construction, à la gestion de votre personnel, etc.

- Sélectionnez-vous personnellement vos collaborateurs? Comment formez-vous votre équipe? Sur quelle base?

- Avez-vous déjà collaboré avec des architectes étrangers? Dans quelles circonstances?

- En termes de processus de conception, y a-t-il une différence entre votre cabinet à Tokyo et celui à Paris?

- Avec un nombre croissant de projets dans différents endroits du monde, quelles sont les procédures que vous mettez en place pour gérer le processus de conception? 